\title{
MODEL REDUCTION VIA MATRIX PENCIL APPROACH
}

Herbert W. Beke

Dept. of Electrical Engineering

University of Minnesota

Minneapolis, MN. 55455

beke@ee.umn.edu
Daniel Boley

Dept. of Computer Science

University of Minnesota

Minneapolis, MN. 55455

boley@cs.umn.edu

Technical Report \# TR 93-45, Dept. of Computer Science, University of Minnesota, Minneapolis, MN. 55455; June 1993.

\begin{abstract}
In this paper, we present a novel way of model reduction based on matrix pencil theory. Using only orthogonal transformations on state space models, we construct the smallest perturbation to the coefficients that yields a lower order system. We derive some bounds on the stability of the resulting lower order system. We illustrate our method with two examples arising from large flexible space structures.
\end{abstract}




\section{Introduction}

Model reduction refers to the approximation of a linear system model by a lower order model such that certain criteria are met. The motivations for model reduction are mainly economical since low order models can be analyzed, simulated or built at a much lower cost than high order models. Numerous papers have been written on model reduction. Reduction by modal expansion appears to be one of the oldest approaches. However, reduction by balanced realization [7] and by optimal Hankel norm approximation [9] as well as their weighted versions [8,10] have now found widespread acceptance among control engineers. In this paper, we present a novel constructive way of implementing model reduction based on some recent results in matrix pencil theory [1]. We now state some definitions and characterizations of matrix pencils $[1,3,4,5]$. We then proceed to relate system theoretic concepts of controllability and observability to matrix pencil theory, and then conclude this introductory section by giving an outline of this paper.

Let $A$ and $B$ be two $n \times p$ matrices. The set of all matrices of the form $(A-\lambda B)$, where $\lambda$ is any complex number is said to be a matrix pencil of dimension $n \times p$. Two matrix pencils $\left(A_{1}-\lambda B_{1}\right)$ and $\left(A_{2}-\lambda B_{2}\right)$ of dimension $n \times p$ are said to be strictly equivalent when there exist constant invertible matrices $P$ and $Q$ of dimension $n \times n$ and $p \times p$ respectively such that $P\left(A_{1}-\lambda B_{1}\right) Q$ $=\left(A_{2}-\lambda B_{2}\right)$. In the classical theory of matrix pencils, it is well known that any pencil is strictly equivalent to its Kronecker Canonical Form (KCF), which is a pseudodiagonal matrix with diagonal blocks of the form $L, L^{T}$, and/or $J$, where

$$
L=\left[\begin{array}{c}
I \\
{[0, \cdots, 0]}
\end{array}\right]+\lambda\left[\begin{array}{c}
{[0, \cdots, 0]} \\
I
\end{array}\right]
$$

is a matrix with one more row than column, and $J$ is a square matrix in Jordan Canonical Form. A matrix pencil $P(\lambda)=(A-\lambda B)$ is said to be deficient if there exists some $\lambda$ for which it is not

full rank. If $P(\lambda)=(A-\lambda B)$ is always full rank for any value of $\lambda$, then it is said to be non-deficient.

We shall consider the standard finite dimensional linear time invariant (FDLTI) continuous time system described by 


$$
\begin{aligned}
& \dot{x}=F x+G u \\
& y=H x
\end{aligned}
$$

where $F \in R^{p \times p}, G \in R^{p \times m}$, and $H \in R^{r \times p}$ as the full order model. $F, G$, and $H$ are also known as the state, input, and output matrices respectively; we have omitted the feedthrough matrix in the state space model description because it remains invariant in the model reduction scheme proposed in this paper. If the full order model is not strictly proper, the method and analysis outlined in this paper can still be applied to the strictly proper part of the model. We shall assume that the system described by (2) is stable, i.e., the real part of all the eigenvalues of $F$ is strictly less than zero. We want to remark that this is a very mild assumption because the transfer function matrix $G(s)$ of any FDLTI system can be decomposed as $G(s)=G_{S}(s)+G_{U}(s)$, where $G_{S}(s)$ is the transfer function matrix of the stable part and $G_{U}(s)$ is the transfer function matrix of the unstable part. One can then proceed with the model order reduction of the stable part. We shall also assume that the state space realization described by (2) is minimal. According to the PBH rank test (see e.g., [4]), the dynamical system described by eqn. (2) is controllable if and only if the matrix pencil

$$
P^{T}(\lambda)=[F-\lambda I \mid G]=[F \mid G]-\lambda[I \mid 0]
$$

has full rank for any complex value $\lambda$. Similarly, the system described by (2) is observable if and only if the matrix pencil

$$
\left[\begin{array}{c}
F-\lambda I \\
H
\end{array}\right]=\left[\begin{array}{l}
F \\
H
\end{array}\right]-\lambda\left[\begin{array}{l}
I \\
0
\end{array}\right]
$$

has full rank for any complex value $\lambda$.

In [1], the sensitivity of an algebraic (Kronecker) structure of rectangular matrix pencils to perturbations in the coefficients was examined and eigenvalue perturbation bounds in the spirit of Bauer-Fike was used to develop computational upper and lower bounds on the distance from a given pencil to one with a qualitatively different Kronecker structure. In this paper, we exploit the bounds derived in [1] for estimating the upper and lower bounds on the distance to a deficient pencil [1], to implement a model reduction scheme. 
The rest of the paper is organized as follows. In section 2, we review the background material on the computation of the nearest distance to a deficient pencil as espoused in [1]. In section 3 , we discuss how the ideas in section 2 can be employed in a model reduction. In section 4 we present an algorithm for model reduction and make some remarks on how it can be modified to suit the particular needs of the designer. In section 5 we address the issue of the stability of the reduced order model. In section 6 we derive the state space expression for the model reduction error in terms of the full order model i.e., (2) and the perturbation of the matrix pencil that leads to a deficient pencil. For a special case of our method, we show in section 5 that the reduced order model is stable so long as the full order model is stable; and in section 6, we derive an upper bound on the $\mathrm{H}_{2}$ norm of the model reduction error for that special case. In section 7, we present some numerical results. Finally, in section 8 , we make some concluding remarks. 


\section{Computation of Deficient Pencil}

In this section we discuss two issues of importance to the reduction scheme as they were espoused in [1]. The first one concerns the problem of determining whether a given rectangular pencil is deficient or not. Specifically, given an $n \times p$ pencil $P(\lambda)=(A-\lambda B)$, with $n>p$, determine whether or not $(A-\lambda B)$ loses rank for any $\lambda$, including possibly $\lambda$ infinite. By augmenting $A$ and $B$ matrices with arbitrary $n \times(n-p)$ matrices $C, D$. We can examine the square $n \times n$ generalized eigenvalue problem

$$
[A, C] v=\lambda[B, D] v
$$

Now, by partitioning the vector $v$ as $v^{T} \equiv\left[x^{T}, y^{T}\right]$, where $x$ is a $p$-vector, and $y$ is a $(n-p)$-vector, the following proposition can be easily derived.

\section{Proposition 1 [Boley].}

Given an $n \times p$ pencil $(A-\lambda B)$ with $n>p$, and given an arbitrary full-rank $n \times(n-p)$ matrices $C, D$, the following are equivalent:

(a) $\quad A-\lambda B$ is a deficient pencil.

(b) Equation (5) has an annihilating vector $v_{0}$ whose last $n-p$ components $y_{0}$ are zero. Call the corresponding annihilating value $\lambda_{0}$.

Furthermore, we have the following:

(c) If $B$ has full column rank, then all the annihilating vectors $v_{0}$ and corresponding values $\lambda_{0}$ are exactly the generalized eigenpairs for the regular part of the pencil.

Proof: See [1].

The issue of choosing $C$ and $D$ has also been addressed in [1] where two choices were proposed for the selection of $C$ and $D$. One of these choices is to select $C$ and $D$ as orthonormal basis of the space orthogonal to the columns of $A$ and $B$. This choice has the effect of limiting the increase to the condition numbers of $[A, C]$ and $[B, D]$ with respect to inversion. Thus in the case $B=\left[I_{p}, 0\right]^{T}, D$ is chosen as $D=\left[0, I_{n-p}\right]^{T}$ to turn the problem into an ordinary eigenvalue problem; also from the $Q-R$ factorization of $A$ we have 


$$
A=\left[Q_{1} Q_{2}\right]\left[\begin{array}{c}
R_{1} \\
0
\end{array}\right],
$$

we choose $C=Q_{2}$. We shall say more about the choice of $C$ in section 4 where we relate the matrix $C$ to the stability of the reduced order model.

The second issue concerns the problem of computing the upper bound on the distance to a deficient pencil. Specifically, consider a nondeficient $n \times p$ pencil $P(\lambda)=(A-\lambda B)$. In this case, we know that $B$ has full rank. We would like to estimate the size of the perturbation $E$ to the matrix $A$ that is needed to obtain a deficient pencil $(A+E-\lambda B)$. In [2], it was shown that the smallest perturbation $E$ can be obtained by solving the minimization problem

$$
\min _{s} \sigma_{\min }(A-s B),
$$

where $\sigma_{\min }(M)$ denotes the smallest singular value of the matrix $M$, and $s$ varies over the entire complex plane. If we denote by $\sigma^{*}$ and $s^{*}$ the minimum in (7) and value of $s$ achieving that minimum, respectively, then $\|E\|=\sigma^{*}$. In [1], it was shown that a simpler scheme which provides both a good estimate for $\|E\|$ and for that value of $s$ that yields the minimum in (7) involves solving the eigenvalue problem (5). Let

$$
\lambda_{i}, v_{i}:=\left[\begin{array}{c}
x_{i} \\
y_{i}
\end{array}\right], \quad i=1, \cdots, n
$$

be the generalized eigenvalues and eigenvectors for (5). For each $i$ we have the equation $[A, C] v_{i}=\lambda_{i}[B, D] v_{i}$, using (8) this equation can be rewritten as $\left(\lambda_{i} B-A\right) x_{i}=\left(C-\lambda_{i} D\right) y_{i}$. For each $i$ define the residual $r_{i}$ as

$$
r_{i}:=\left(A-\lambda_{i} B\right) x_{i} ;
$$

and the perturbation $E_{i}$ as

$$
E_{i}:=\frac{-r_{i} x_{i}{ }^{T}}{\left\|x_{i}\right\|^{2}} \equiv\left(\lambda_{i} B-A\right) \frac{x_{i} x_{i}{ }^{T}}{\left\|x_{i}\right\|^{2}}=\left(C-\lambda_{i} D\right) \frac{y_{i}}{\left\|x_{i}\right\|} \cdot \frac{x_{i}{ }^{T}}{\left\|x_{i}\right\|} .
$$

We remark that the norm on $E_{i}$ can be simply computed as 


$$
\left\|E_{i}\right\|=\frac{\left\|r_{i}\right\|_{2}}{\left\|x_{i}\right\|_{2}} .
$$

Note that $E_{i} x_{i}=-r_{i}$, and thus (9) becomes

$$
\left(A+E_{i}-\lambda_{i} B\right) x_{i}=0 .
$$

Now, $\left(A+E_{i}-\lambda B\right)$ is a deficient pencil, losing rank exactly at $\lambda=\lambda_{i}$, for each $i$. Let $\sigma_{i}, u_{i}, w_{i}$ be, respectively, the smallest singular value and the corresponding left and right singular vectors of $\left(A-\lambda_{i} B\right)$ for each $i$. Then $E_{i}^{\prime}:=-\sigma_{i} u_{i} w_{i}^{T}$ is another smaller perturbation yielding a deficient pencil. By taking norms of (10), the following bounds are obtained for the perturbations: $\left\|E_{i}^{\prime}\right\|$ $\leq\left\|E_{i}\right\| \leq\left\|\left(C-\lambda_{i} D\right) y_{i}\right\| /\left\|x_{i}\right\|$. We also have the following error bound from [1]. If $E$ denotes that perturbation with the smallest norm yielding a deficient pencil, then $E$ satisfies

$$
\begin{aligned}
\|E\| & \equiv \sigma^{*} \leq \beta_{2} \equiv \min _{i}\left\|E_{i}^{\prime}\right\| \equiv \min _{i} \sigma_{\min }\left(A-\lambda_{i} B\right) \\
& \leq \beta_{1} \equiv \min _{i}\left\|E_{i}\right\| \equiv \min _{i} \frac{\left\|\left(C-\lambda_{i} D\right) y_{i}\right\|}{\mid x_{i} \|}
\end{aligned}
$$

The importance of this error bound was noted and stated as the following proposition in [1].

\section{Proposition 2 [Boley].}

Let $(A-\lambda B)$ be an $n \times p$ pencil, with $n>p$. Let $C, D$ be two arbitrary full-rank $n \times(n-p)$ matrices. Then the smallest perturbation $E$ such that $A+E-\lambda B$ is a deficient pencil satisfies the bound (13), where $\lambda_{i}, v_{i}, i=1, \cdots, n$ are the eigenpairs of the generalized eigenproblem (5) and $y_{i}$ are defined by (8).

Proof. See [1]. 


\section{Application to Model Reduction}

Our goal is to use the results of the previous section to implement a model reduction scheme. This is done by constructing a matrix pencil based on the PBH test for controllability or observability. The matrix pencil so constructed is augmented with " $C$ " and " $D$ " matrices as described in section 2. One then proceeds to solve the eigenvalue problem which is (5). Next, the norm of the perturbations $\left\|E_{i}\right\|, i, \cdots, n$, are computed using (11) and sorted in increasing order. Using the eigenpair $\left(\lambda_{i}, v_{i}\right)$ corresponding to the smallest value of $\left\|E_{i}\right\|$, one constructs a perturbation matrix $E_{i}$ such that $\left(A+E_{i}-\lambda B\right)$ is a deficient pencil, losing rank exactly at $\lambda=$ $\lambda_{i}$. The resulting uncontrollable or unobservable state is then truncated to yield a reduced order system. The method is then extended so that eigenpairs $\left(\lambda_{i}, v_{i}\right), i=1, \cdots, N$ corresponding to the first $N$ smallest value of $\left\|E_{i}\right\|$, can be used to construct a perturbation matrix $E_{i}$ such that $\left(A+E_{i}-\lambda B\right)$ is a deficient pencil, losing rank exactly at $\lambda=\lambda_{i}$, for each $i=1, \cdots, N$. The resulting uncontrollable or unobservable states are then truncated to yield a reduced order system.

\subsection{Reduction by a Single Mode Using Controllability Criteria}

Here, we shall assume that the $A$ and $B$ matrices of the matrix pencil $P(\lambda)=(A-\lambda B)$ are defined by

$$
A:=\left[\begin{array}{l}
F^{T} \\
G^{T}
\end{array}\right] \quad B:=\left[\begin{array}{c}
I_{p} \\
0
\end{array}\right]
$$

where the underlying matrices $F$ and $G$ are given by (2). When the $A$ and $B$ matrices are formed as above we shall refer to the matrix pencil $P(\lambda)=(A-\lambda B)$ as being formed from the controllability condition. Suppose the perturbation matrix $E_{i}$ is partitioned as $E_{i}^{T}=\left[E_{1}, E_{2}\right]$ where $E_{1}$ and $E_{2}$ have the dimensions of $F$ and $G$ respectively. Substituting for the $A, B$, and $E_{i}$ matrices in (12), we obtain 


$$
\left[\left(\begin{array}{l}
F^{T}+E_{1}{ }^{T} \\
G^{T}+E_{2}{ }^{T}
\end{array}\right)-\lambda_{i}\left(\begin{array}{c}
I_{p} \\
0
\end{array}\right)\right] x_{i}=0 ;
$$

We now look at the two set of equations in (15). The top part yields $\left(F+E_{1}\right)^{T} x_{i}=\lambda_{i} x_{i}$. When this is premultiplied by a Householder transformation $Q$ such that $Q x_{i}=e_{1}$, where $e_{1}$ is the first column of the identity matrix, we obtain

$$
Q\left(F+E_{1}\right)^{T} Q^{T} e_{1}=\lambda_{i} Q x_{i}=\lambda_{i} e_{1},
$$

which has the following structure

$$
\left[\begin{array}{cccc}
\lambda_{i} & x & \cdots & x \\
0 & x & \cdots & x \\
\vdots & \vdots & \ddots & \vdots \\
0 & x & \cdots & x
\end{array}\right]\left(\begin{array}{l}
1 \\
0 \\
\vdots \\
0
\end{array}\right)=\lambda_{i}\left(\begin{array}{c}
1 \\
0 \\
\vdots \\
0
\end{array}\right)
$$

Similarly, the bottom part of (15) yields $\left(G+E_{2}\right)^{T} x_{i}=0$. When this is transposed, we obtain

$$
x_{i}^{T} Q{ }^{T} Q\left(G+E_{2}\right)=e_{1}^{T} Q\left(G+E_{2}\right)=0 \text {, }
$$

which also has the following structure

$$
\left(\begin{array}{llll}
1 & 0 & \cdots & 0
\end{array}\right)\left[\begin{array}{ccc}
0 & \cdots & 0 \\
x & \cdots & x \\
\vdots & \ddots & \vdots \\
x & \cdots & x
\end{array}\right]=\left(\begin{array}{lll}
0 & \cdots & 0
\end{array}\right)
$$

The perturbed system can be written as

$$
\begin{aligned}
& \dot{z}=Q\left(F+E_{1}\right) Q^{T} z+Q\left(G+E_{2}\right) u \\
& y=H Q^{T} z
\end{aligned}
$$

where $Q\left(F+E_{1}\right) Q^{T}$ and $Q\left(G+E_{2}\right)$ have the structures shown below 


$$
Q\left(F+E_{1}\right) Q^{T}=\left[\begin{array}{cccc}
\lambda_{i} & 0 & \cdots & 0 \\
x & x & \cdots & x \\
\vdots & \vdots & \ddots & \vdots \\
x & x & \cdots & x
\end{array}\right], \quad Q\left(G+E_{2}\right)=\left[\begin{array}{ccc}
0 & \cdots & 0 \\
x & \cdots & x \\
\vdots & \ddots & \vdots \\
x & \cdots & x
\end{array}\right] .
$$

Clearly, the first state in the transformed state $z$ is not controllable. If we denote

$$
Q\left(F+E_{1}\right) Q^{T}, Q\left(G+E_{2}\right), H Q^{T} \text { by } \tilde{F}, \tilde{G} \text {, and } \tilde{H}
$$

respectively, the perturbed system described by eqn. (20) can be written in partitioned form as

$$
\begin{aligned}
{\left[\begin{array}{l}
\dot{z}_{1} \\
\dot{z}_{2}
\end{array}\right] } & =\left[\begin{array}{cc}
\tilde{F}_{11} & 0 \\
\tilde{F}_{21} & \tilde{F}_{22}
\end{array}\right]\left[\begin{array}{l}
z_{1} \\
z_{2}
\end{array}\right]+\left[\begin{array}{c}
0 \\
\tilde{G}_{2}
\end{array}\right] u \\
y & =\left[\begin{array}{ll}
\tilde{H}_{1} & \tilde{H}_{2}
\end{array}\right]\left[\begin{array}{l}
z_{1} \\
z_{2}
\end{array}\right] .
\end{aligned}
$$

If the uncontrollable state is stable it can be directly truncated to yield a reduced order model

$$
\begin{aligned}
\dot{z}_{2} & =\tilde{F}_{22} z_{2}+\tilde{G}_{2} u \\
y & =\tilde{H}_{2} z_{2} .
\end{aligned}
$$




\subsection{Reduction by Multiple Modes Using Controllability Criteria}

In general, solving the eigenvalue equation (5) may yield real eigenvalues and complex eigenvalues which occur as complex conjugates. When the perturbation matrix $E_{i}$ corresponds to complex eigenvalues then the reduction scheme needs be slightly altered so that the two states corresponding to the complex eigenvalues are simultaneously truncated. Also, for a large order system, it will be more economical to reduce the order of the system by multiple states instead of a single state each time the matrix pencil is perturbed. In this subsection, we show how to extend our method to handle both situations.

We shall assume that we want to truncate $N$ modes which correspond to the $N$ smallest $\left\|E_{i}\right\|$ values. From the eigenvalues $\lambda_{i}=\alpha_{i}+j \beta_{i}$, with the corresponding eigenvectors $v_{i}=\left[x_{i}^{H}, y_{i}^{H}\right]^{H}$, $i=1, \cdots, N$, we form the matrices $T, W$ and $U$ as follows:

$$
\begin{aligned}
T:=\left[\begin{array}{cccccccc}
\alpha_{1} & \beta_{1} & 0 & 0 & \ldots & \ldots & \ldots & 0 \\
-\beta_{1} & \alpha_{1} & 0 & 0 & \ldots & \ldots & \ldots & 0 \\
0 & 0 & \alpha_{2} & \beta_{2} & 0 & \ldots & \ldots & 0 \\
0 & 0 & -\beta_{2} & \alpha_{2} & 0 & \ldots & \ldots & 0 \\
\vdots & \vdots & 0 & 0 & \ddots & \ddots & \ldots & \vdots \\
\vdots & \vdots & \vdots & \vdots & \ddots & \ddots & 0 & 0 \\
\vdots & \vdots & \vdots & \vdots & \vdots & 0 & \alpha_{N} & \beta_{N} \\
0 & 0 & 0 & 0 & \ldots & 0 & -\beta_{N} & \alpha_{N}
\end{array}\right] \\
W:=\left[\operatorname{Re}\left(x_{1}\right), \operatorname{Im}\left(x_{1}\right), \operatorname{Re}\left(x_{2}\right), \operatorname{Im}\left(x_{2}\right), \cdots, \operatorname{Re}\left(x_{N}\right), \operatorname{Im}\left(x_{N}\right)\right] \\
U:=\left[\operatorname{Re}\left(y_{1}\right), \operatorname{Im}\left(y_{1}\right), \operatorname{Re}\left(y_{2}\right), \operatorname{Im}\left(y_{2}\right), \cdots, \operatorname{Re}\left(y_{N}\right), \operatorname{Im}\left(y_{N}\right)\right]
\end{aligned}
$$

That is the columns of $V=\left[W^{H}, U^{H}\right]^{H}$ form a real basis for the invariant subspace corresponding to the eigenvalues in $T$. The corresponding eigenspace equation (cf. eqn. (5)) becomes 


$$
[A, C]\left[\begin{array}{l}
W \\
U
\end{array}\right]=\left[\begin{array}{c}
W \\
U
\end{array}\right] T \equiv\left[\begin{array}{c}
W T \\
0
\end{array}\right]+\left[\begin{array}{c}
0 \\
U T
\end{array}\right],
$$

We want to remark that actually any basis for the invariant subspace corresponding to the $N$ modes to truncate will do, whether or not they are complex. In such a case, $T$ may be full but will have the same eigenvalues. The following development carries through almost unchanged.

Equation (27) simplifies to

$$
A W-\left[\begin{array}{c}
W T \\
0
\end{array}\right]=\left[\begin{array}{c}
0 \\
U T
\end{array}\right]-C U .
$$

Define the residual $R$ as $R:=$ RHS of (28), and from $E_{i} W+R=0$, define $E_{i}$ as $E_{i}=-R\left(W^{T} W\right)^{-1} W^{T}$. The perturbed pencil equation becomes

$$
\left[A+E_{i}\right] W=\left[\begin{array}{c}
W T \\
0
\end{array}\right] .
$$

Substituting for the $A$ and $E_{i}$ matrices, the equation above becomes

$$
\left[\begin{array}{c}
F^{T}+E_{1}^{T} \\
G^{T}+E_{2}^{T}
\end{array}\right] \cdot W=\left[\begin{array}{c}
W T \\
0
\end{array}\right] .
$$

We now look at the two set of equations in (30). The top part yields $\left(F+E_{1}\right)^{T} W=W T$. Now choose an orthonormal $Q$ such that

$$
Q W=\left[\begin{array}{l}
S \\
0
\end{array}\right]
$$

where 


$$
S=\left[\begin{array}{cccccc}
x & x & \cdots & \cdots & x & x \\
0 & x & \cdots & \cdots & x & x \\
0 & 0 & \ddots & \ddots & \vdots & x \\
0 & 0 & 0 & \ddots & x & x \\
\vdots & \vdots & \ddots & 0 & x & x \\
0 & 0 & \cdots & \cdots & 0 & x
\end{array}\right] .
$$

Thus when the top part of (30) is premultiplied by $Q$, we have $Q\left(F+E_{1}\right)^{T} Q^{T} Q W=Q W T$ which has the following structure upon substituting for $Q W$

$$
Q\left(F+E_{1}\right)^{T} Q^{T}\left[\begin{array}{l}
S \\
0
\end{array}\right]=\left[\begin{array}{c}
S T \\
0
\end{array}\right] .
$$

Similarly, the bottom part of (30) yields $\left(G+E_{2}\right)^{T} Q^{T} Q W=0$. The perturbed system can be written as (20). However, the matrices $Q\left(F+E_{1}\right) Q^{T}$ and $Q\left(G+E_{2}\right)$ take the structures shown below

$$
Q\left(F+E_{1}\right) Q^{T}=\left[\begin{array}{cccccccccc}
x & x & 0 & 0 & \cdots & \cdots & \cdots & 0 & \cdots & 0 \\
x & x & 0 & 0 & \cdots & \cdots & \cdots & 0 & \cdots & 0 \\
x & x & x & x & 0 & \cdots & \cdots & 0 & \cdots & 0 \\
x & x & x & x & 0 & \cdots & \cdots & 0 & \cdots & 0 \\
\vdots & \vdots & \vdots & \vdots & \ddots & 0 & 0 & 0 & \cdots & 0 \\
\vdots & \vdots & \vdots & \vdots & \ddots & x & x & 0 & \cdots & 0 \\
\vdots & \vdots & \vdots & \vdots & \vdots & x & x & 0 & \cdots & 0 \\
x & x & x & x & \cdots & x & x & x & \cdots & x \\
\vdots & \vdots & \vdots & \vdots & \ddots & \vdots & \vdots & \vdots & \vdots & \vdots \\
x & x & x & x & \cdots & x & x & x & \cdots & x
\end{array}\right], \quad Q\left(G+E_{2}\right)=\left[\begin{array}{ccc}
0 & \cdots & 0 \\
0 & \cdots & 0 \\
\vdots & \ddots & \vdots \\
\vdots & & \vdots \\
\vdots & & \vdots \\
\vdots & \cdots & \vdots \\
0 & \cdots & 0 \\
x & \cdots & x \\
\vdots & \ddots & \vdots \\
x & \cdots & x
\end{array}\right] .
$$

Clearly the first $2 N$ states in the transformed coordinate system are not controllable. One can write down the equation of the perturbed system as eqn. (23) where $z_{1}$ represents the uncontrollable $2 N$ states. If the uncontrollable $2 N$ states are stable, then by direct truncation of the states $z_{1}$, a reduced order model which is described by eqn. (23) can be obtained. 


\subsection{Reduction by a Single Mode Using Observability Criteria}

Here we shall assume that the $A$ and $B$ matrices of the matrix pencil $P(\lambda)=(A-\lambda B)$ are defined by

$$
A:=\left[\begin{array}{l}
F \\
H
\end{array}\right] \quad B:=\left[\begin{array}{l}
I_{p} \\
0
\end{array}\right]
$$

where the underlying matrices $F$ and $H$ are given by (2). When the $A$ and $B$ matrices are formed as above we shall refer to the matrix pencil $P(\lambda)=(A-\lambda B)$ as being formed from the observability condition. The perturbation matrix $E_{i}$ is partitioned as $E_{i}^{T}=\left[E_{1}{ }^{T}, E_{2}^{T}\right]$ where $E_{1}$ and $E_{2}$ are partitioned to have the dimensions of $F$ and $H$ respectively. Substituting for the $A, B$, and $E_{i}$ matrices in (12), yields

$$
\left[\left(\begin{array}{l}
F+E_{1} \\
H+E_{2}
\end{array}\right)-\lambda_{i}\left(\begin{array}{l}
I_{p} \\
0
\end{array}\right)\right] x_{i}=0 .
$$

We now look at the two set of equations in (36). The top part yields $\left(F+E_{1}\right) x_{i}=\lambda_{i} x_{i}$. When this is premultiplied by a Householder transformation $Q$ such that $Q x_{i}=e_{1}$, we obtain

$$
Q\left(F+E_{1}\right) Q^{T} e_{1}=\lambda_{i} Q x_{i}=\lambda_{i} e_{1},
$$

which has the following structure

$$
\left[\begin{array}{cccc}
\lambda_{i} & x & \cdots & x \\
0 & x & \cdots & x \\
\vdots & \vdots & \ddots & \vdots \\
0 & x & \cdots & x
\end{array}\right]\left(\begin{array}{l}
1 \\
0 \\
\vdots \\
0
\end{array}\right)=\lambda_{i}\left(\begin{array}{c}
1 \\
0 \\
\vdots \\
0
\end{array}\right) .
$$

Similarly, the bottom part of (36) yields $\left(H+E_{2}\right) Q^{T} Q x_{i}=0$. Since $Q x_{i}=e_{1}$, this equation can be written symbolically as: 


$$
\left[\begin{array}{cccc}
0 & x & \cdots & x \\
\vdots & \vdots & \ddots & \vdots \\
0 & x & \cdots & x
\end{array}\right]\left(\begin{array}{c}
1 \\
0 \\
\vdots \\
0
\end{array}\right)=\left(\begin{array}{c}
0 \\
0 \\
\vdots \\
0
\end{array}\right)
$$

The perturbed system can be written as

$$
\begin{aligned}
& \dot{z}=Q\left(F+E_{1}\right) Q^{T} z+Q G u \\
& y=\left(H+E_{2}\right) Q^{T} z,
\end{aligned}
$$

where $Q\left(F+E_{1}\right) Q^{T}$ and $\left(H+E_{2}\right) Q^{T}$ have the structures shown below

$$
\begin{aligned}
Q\left(F+E_{1}\right) Q^{T} & =\left[\begin{array}{cccc}
\lambda_{i} & x & \cdots & x \\
0 & x & \cdots & x \\
\vdots & \vdots & \ddots & \vdots \\
0 & x & \cdots & x
\end{array}\right] \\
\left(H+E_{2}\right) Q^{T} & =\left[\begin{array}{cccc}
0 & x & \cdots & x \\
\vdots & \vdots & \ddots & \vdots \\
0 & x & \cdots & x
\end{array}\right] .
\end{aligned}
$$

Clearly, the first mode in the transformed state $z$ is not observable. If we denote

$$
Q\left(F+E_{1}\right) Q^{T}, Q G,\left(H+E_{2}\right) Q^{T} \text { by } \tilde{F}, \tilde{G}, \text { and } \tilde{H}
$$

respectively, the perturbed system described by (40) can be written in partitioned form as

$$
\begin{aligned}
{\left[\begin{array}{l}
\dot{z}_{1} \\
\dot{z}_{2}
\end{array}\right]=\left[\begin{array}{cc}
\tilde{F}_{11} & \tilde{F}_{12} \\
0 & \tilde{F}_{22}
\end{array}\right]\left[\begin{array}{l}
z_{1} \\
z_{2}
\end{array}\right]+\left[\begin{array}{c}
\tilde{G}_{1} \\
\tilde{G}_{2}
\end{array}\right] u } \\
y=\left[\begin{array}{ll}
0 & \tilde{H}_{2}
\end{array}\right]\left[\begin{array}{l}
z_{1} \\
z_{2}
\end{array}\right] .
\end{aligned}
$$

If the unobservable mode is stable it can be directly truncated to yield a reduced order model 


$$
\begin{aligned}
& \dot{z}_{2}=\tilde{F}_{22} z_{2}+\tilde{G}_{2} u \\
& y=\tilde{H}_{2} z_{2} .
\end{aligned}
$$

\subsection{Reduction by Multiple Modes Using Observability Criteria}

The procedure here is similar to that discussed in subsection 3.2. Using the ideas presented in subsections 3.2 and 3.3, one can easily write down the procedure for truncating multiple modes using the observability condition.

\subsection{On the Choice of Controllability or Observability Criteria}

The trace of the controllability and observability grammians give a measure of how "controllable" or how "observable" the model is in its current state space realization. Denote by $\kappa_{C}$ the norm of the perturbation to the input matrix $G$ that will render the realization uncontrollable, and $\kappa_{O}$ the norm of the perturbation to the output matrix $H$ that will render the same realization unobservable. We shall also denote the controllability and observability grammians by $W_{\text {contr }}$ and $W_{o b s}$ respectively. It is desirable to effect the model reduction by applying the smallest possible perturbation. If trace $\left(W_{c o n t r}\right)<\operatorname{trace}\left(W_{o b s}\right)$ then we normally expect to have $\kappa_{C}<\kappa_{O}$, in that case the controllability method should be used. On the other hand if $\operatorname{trace}\left(W_{\text {contr }}\right)>\operatorname{trace}\left(W_{\text {obs }}\right)$ then we normally expect to have $\kappa_{C}>\kappa_{O}$, and in that case the observability method should be used. 


\section{Algorithm for Model Reduction}

Given a FDLTI system as described by equation (2), a reduced order model can be computed using the following algorithm.

Step 1

$\operatorname{Compute} \operatorname{trace}\left(W_{\text {contr }}\right)$ and $\operatorname{trace}\left(W_{\text {obs }}\right)$. If $\operatorname{trace}\left(W_{\text {contr }}\right)<\operatorname{trace}\left(W_{\text {obs }}\right)$ then use the $\mathrm{PBH}$ controllability test to form the matrix pencil as described in equation (3). Otherwise use the PBH observability test to form the matrix pencil. Augment the pencil with $C$ and $D$ matrices as described in section 2 .

Step 2

Solve the corresponding eigenvalue problem which is (5). Next, compute $\left\|E_{i}\right\|, i=1, \cdots, n$, and sort them in increasing order. As remarked in section 2, $\left\|E_{i}\right\|$ can be computed as $\left\|E_{i}\right\|=\left\|r_{i}\right\|_{2} /\left\|x_{i}\right\|_{2}$, and in the case of complex eigenvalues, one need compute $\left\|E_{i}\right\|$ only once for the pair of complex conjugates. Also identify all the spurious eigenvalues.

Step 3

Make a decision on the number of states the reduced order model must have. Construct the perturbation matrix $E_{i}$ as described in section 2 or 3 using the eigenpairs corresponding to the smallest values of $\left\|E_{i}\right\|$. Next form the perturbed system as described in section 3 and then apply the appropriate orthonormal transformation matrix $Q$ to the perturbed system to reveal the uncontrollable or unobservable states. Verify the stability of the perturbed system. A reduced order model can be obtained by direct truncation of the resulting uncontrollable or unobservable states which are stable.

\section{Remarks}

1. The algorithm outlined above may be modified to suit particular needs of the designer. For example, if $\operatorname{trace}\left(W_{c o n t r}\right)<\operatorname{trace}\left(W_{o b s}\right)$ but close in value, one may use the controllability method to truncate a few states and then implement the observability method to truncate other states.

2. The algorithm proposed above could be extended to include frequency dependent 
weightings. The modes of the full order model can be weighted differently and a different criterion can be used for truncation. For example, one could use a weighted average of the existing measure and the maximum singular values of the transfer function matrix . For simplicity, we have not done that here.

3. The method presented in this paper involves using an orthonormal matrix $Q$ to effect a similarity transformation. Model reduction by optimal Hankel norm reduction [9] and by truncation by balanced realization [7] also involve using a matrix $T$ to effect a similarity transformation, however, the $T$ used in [7,9] is not in general an orthonormal matrix. As is well known, use of non-orthonormal (or non-unitary) transformation can lead to worsening of the condition numbers and loss of accuracy of the system matrices. Because of this fact, the method presented in this paper is at a significant advantage over the methods of $[7,9]$. 


\section{Stability of the Reduced Order Model}

\subsection{Stability of the Reduced Order Model for Continuous Time FDLTI System}

A question of great importance in any model reduction scheme is whether the reduced order model obtained from a stable full order model is stable or not. In our scheme, this amounts to asking whether the real part of all the eigenvalues of $Q\left(F+E_{1}\right) Q^{T}$ are strictly less than zero. First of all we note that $\left(F+E_{1}\right)$ and $Q\left(F+E_{1}\right) Q^{T}$ are similar matrices; therefore they have the same eigenvalues with the same multiplicities. Consequently, it will suffice to consider the eigenvalues of $\left(F+E_{1}\right)$. We shall focus on the controllability condition, however the discussions concerning the stability of $\left(F+E_{1}\right)$ are equally valid for the observability condition.

In order to form the perturbation matrix $E_{i}$, we solve the eigenvalue equation $[A, C] v_{i}=\lambda_{i}[B, D] v_{i}$, where $A$ is formed as $A=[F, G]^{T}, B=\left[I_{p}, 0\right]^{T}$ and set $D=\left[0, I_{n-p}\right]^{T}$ to turn the problem into an ordinary eigenvalue problem. There is some flexibility in the choice of $C$, however in this paper we shall form $C$ as discussed in section 2, i.e., we select $C$ as orthonormal basis of the space orthogonal to the columns of $A$. Furthermore, we partition the matrix $C$ as $C^{T}=\left[C_{1}{ }^{T}, C_{2}{ }^{T}\right]$, where $C_{1}$ and $C_{2}$ are of dimensions $p \times(n-p)$ and $(n-p) \times(n-p)$ respectively. We also introduce the parameter $\alpha$ by writing the matrix $C$ as

$$
C=\left[\begin{array}{c}
\alpha C_{1} \\
C_{2}
\end{array}\right] ; \quad \alpha \in[0,1]
$$

Then the eigenvalue equation can be written as

$$
\left[\begin{array}{cc}
F^{T} & \alpha C_{1} \\
G^{T} & C_{2}
\end{array}\right]\left[\begin{array}{l}
x_{i} \\
y_{i}
\end{array}\right]=\lambda\left[\begin{array}{l}
x_{i} \\
y_{i}
\end{array}\right],
$$

which can be manipulated into the form

$$
\left[\begin{array}{l}
F^{T} \\
G^{T}
\end{array}\right] x_{i}-\lambda_{i}\left[\begin{array}{l}
x_{i} \\
0
\end{array}\right]=\lambda\left[\begin{array}{l}
0 \\
y_{i}
\end{array}\right]-\left[\begin{array}{l}
\alpha C_{1} \\
C_{2}
\end{array}\right] y_{i}=\left[\begin{array}{l}
r_{1} \\
r_{2}
\end{array}\right] .
$$

We defined the RHS of eqn. (47) as the residual vector $r_{i}$, which is partitioned as $r_{i}^{T}=\left[r_{1}^{T}, r_{2}^{T}\right]$. We also partitioned the perturbation matrix $E_{i}$ conformably as $E_{i}^{T}=\left[E_{1}, E_{2}\right]$. In eqn. (10), $E_{i}$ is 
defined as $-r_{i} x_{i}^{T} /\left\|x_{i}\right\|$, it can be written in partitioned form as

$$
\left[\begin{array}{l}
E_{1} \\
E_{2}
\end{array}\right]=-\left[\begin{array}{l}
r_{1} \\
r_{2}
\end{array}\right] \frac{x_{i}{ }^{T}}{x_{i}{ }^{T} x_{i}} .
$$

When $\alpha=0$, then $r_{1}$ is a zero vector and $E_{1}$ is a zero matrix which implies there is no perturbation of the $F$ matrix. In that case the reduced order model remains stable so long as the original model is stable. As $\alpha$ increases, $E_{1}$ is no longer zero but varies continuously with $\alpha$. Therefore stability of $\left(F+E_{1}\right)$ is maintained for small values of $\alpha$. We derive below some bounds on $\alpha$ that maintain stability, though in practice we found it just sufficed to verify the stability of the perturbed system.

A matrix is defined to be unstable if it has at least one eigenvalue in the open right half plane (RHP). We may define a matrix to be neutrally stable if all eigenvalues on the imaginary axis are simple and no eigenvalue lies in the open RHP. To derive bounds on $\alpha$ that maintain stability, we need to measure how close the system matrix $F$ is to neutral stability so that we can estimate limit on $\alpha$ to ensure that $\left(F+E_{1}\right)$ remains stable. We shall use the Bauer-Fike theorem which can be stated as follows.

\section{Theorem 1 (Bauer-Fike theorem):}

We are given an $n \times n$ matrix $A$ with a complete set of eigenvalues $\lambda_{1}, \cdots, \lambda_{n}$ and corresponding left and right eigenvectors $w_{1}, \cdots, w_{n}, v_{1}, \cdots, v_{n}$. Let $V:=\left[v_{1}, \cdots, v_{n}\right]$ be the matrix of eigenvectors. Let $\Delta$ be another arbitrary $n \times n$ matrix, and let $\mu$ be any eigenvalue of $A+\Delta$. Let $K$ be the condition number of $V$, i.e., $K=\|V\|\left\|V^{-1}\right\|$. Then for at least one $\lambda_{i}, 1 \leq i \leq n$, the following bound holds:

$$
\left|\mu-\lambda_{i}\right| \leq K\|\Delta\| \text {. }
$$

Proof: See [6]

Application of the theorem gives us the following result. Let $K$ be the condition number of the eigenvectors of $F$ and $d$ the distance to the imaginary axis of the closest eigenvalue of $F$ to the imaginary axis. Then stability of $\left(F+E_{1}\right)$ is guaranteed as long as 


$$
K\left\|E_{1}\right\|<d .
$$

We want to remark that the bound on the eigenvalue perturbation as given in (50) is quite a conservative estimate. Suppose we define $d_{i}$ to be the distance to the imaginary axis of the $i$-th eigenvalue of $F$, and $K_{i}$ to be the smaller of $K$ and the condition number of the $i$-th eigenvector of $F$ [1], i.e.,

$$
K_{i} \equiv \min \left(\|V\| \cdot\left\|V^{-1}\right\|, \frac{n}{s_{i}}\right) \quad \text { with } \quad s_{i} \equiv \frac{\left|w_{i}{ }^{H} v_{i}\right|}{\left\|w_{i}\right\| \cdot\left\|v_{i}\right\|} \text {. }
$$

If $K$ is replaced by the individual $K_{i}$ and $d$ by the individual $d_{i}$, then stability of $\left(F+E_{1}\right)$ is guaranteed as long as

$$
K_{i} \mid E_{1} \|<d_{i}, \quad \forall i .
$$

This will yield a slightly tighter bound.

\subsection{Stability of a Reduced Order Model for a Discrete Time FDLTI System}

The model reduction scheme outlined in section 3 of this paper can be applied with very little modification to the discrete time FDLTI described by

$$
\begin{aligned}
x(k+1) & =F x(k)+G u(k) \\
y(k) & =H x(k),
\end{aligned}
$$

with the same assumptions as stated for the continuous time system on page 2. However, the stability tests outlined in equations (50) and (52) need to be modified. The discrete time system is said to be stable if all the eigenvalues of $F$ are within the open unit circle. Consequently for the discrete time system, a matrix is defined to be unstable if it has at least one eigenvalue outside the closed unit circle. We may also define a matrix to be neutrally stable if all eigenvalues on the unit circle are simple and no eigenvalue lies outside the closed unit circle. Equations (50) and (52) can then be used with $d$ defined as the distance to the unit circle of the closest eigenvalue of $F$ to the unit circle, and $d_{i}$ as the distance to the unit circle of the $i$-th eigenvalue of $F$. 


\subsection{Spurious Eigenvalues}

Another issue of concern is that of the $n-p$ spurious eigenvalues introduced when the eigenvalue equation is solved. These spurious eigenvalues arise because the underlying dynamical system of the matrix pencil is of order $p$ whereas the eigenvalue equation yields $n$ eigenvalues. Note that when $\alpha=0$, the spurious eigenvalues pose no problem because the vector $x_{i}=0$, thus $\left\|E_{i}\right\|=\infty$ for the spurious eigenvalues and hence can be ignored in the model reduction process. However, when $\alpha \neq 0$ the value of $\left\|E_{i}\right\|$ for the spurious roots is finite. Generally, $\left\|E_{i}\right\|$ for the spurious eigenvalues will be much larger than for the non-spurious eigenvalues. To see this, consider the PBH eigenvector test.

\section{Theorem 2 (PBH Eigenvector Test):}

The pair $\{F, G\}$ is noncontrollable if and only if there exists a row vector $q \neq 0$ such that

$$
q F=\lambda q, \quad q G=0
$$

i.e., $\{F, G\}$ is controllable if and only if there is no row vector or left eigenvector of $F$ that is orthogonal to $G$.

Proof: See [4]

Let $\left\|E_{i}\right\|=\varepsilon$, where $\varepsilon$ is a finite nonzero value; this can be stated as

$$
\left\|E_{i}\right\|=\frac{1}{\left\|x_{i}\right\|}\left\|r_{1}\right\|=\varepsilon .
$$

Thus we must have $\left\|r_{1}\right\| /\left\|x_{i}\right\| \leq \varepsilon$ and $\left\|r_{2}\right\| /\left\|x_{i}\right\| \leq \varepsilon$. From eqn. (47), the residual vector components can be written as

$$
r_{1}^{T}=x_{i}^{T}\left(F-\lambda_{i} I\right), \quad \text { and } \quad r_{2}^{T}=x_{i}^{T} G
$$

Now $\left\|r_{1}\right\| /\left\|x_{i}\right\|$ small implies the pair $\left(\lambda_{\mathrm{i}}, x_{i}^{T}\right)$ is almost an eigenpair of $F$ and in light of the PBH eigenvector test, $\left\|r_{2}\right\| /\left\|x_{i}\right\|$ small implies the pair $\{F, G\}$ is nearly uncontrollable. On the other hand $\left\|r_{1}\right\| /\left\|x_{i}\right\|$ large implies that $\lambda_{i}$ probably does not correspond to any eigenvalue of $F$ and 
hence is spurious. Consequently, we expect the $\left\|E_{i}\right\|$ values for the spurious eigenvalues to be larger than the $\left\|E_{i}\right\|$ values for the approximate eigenvalues of $F$. In the numerical tests that we conducted, we observed that when the computed $\left\|E_{i}\right\|$ values are sorted in increasing order then

1) there is always a discernable gap between the $p$-th and the $(p+1)$-th values and 2) the last $n-p$ values of $\left\|E_{i}\right\|$ correspond to the spurious eigenvalues. 


\section{Model Reduction Error}

In this section we derive state space expressions for the model reduction error. We shall assume that the reduced order model was obtained using the controllability condition. By a slight modifications of the equations below one can obtain similar expressions for a reduced order model obtained using the observability condition. We consider two cases which are 1) $\alpha=0$, and 2) $\alpha \neq 0$.

When $\alpha=0$, then $E_{1}$ is a zero matrix which implies there is no perturbation of the $F$ matrix and it is only the $G$ matrix which is perturbed. If we apply the orthonormal matrix $Q$ (from eqn. (31)) to the state space representation of the full order model $G(s)$ (eqn. (2)), we have the following

$$
\begin{aligned}
& \dot{z}=Q F Q^{T} z+Q G u \\
& y=H Q^{T} z
\end{aligned}
$$

Denote

$$
Q F Q^{T}, Q G, H Q^{T} \text { by } \hat{F}, \Gamma, \text { and } \hat{H}
$$

respectively; the similarity transformed system described by eqn. (57) can be written in partitioned form as

$$
\begin{aligned}
{\left[\begin{array}{c}
\dot{z}_{1} \\
\dot{z}_{2}
\end{array}\right] } & =\left[\begin{array}{ll}
\hat{F}_{11} & 0 \\
\hat{F}_{21} & \hat{F}_{22}
\end{array}\right]\left[\begin{array}{l}
z_{1} \\
z_{2}
\end{array}\right]+\left[\begin{array}{l}
\Gamma_{1} \\
\Gamma_{2}
\end{array}\right] u \\
y & =\left[\begin{array}{ll}
\hat{H}_{1} & \hat{H}_{2}
\end{array}\right]\left[\begin{array}{l}
z_{1} \\
z_{2}
\end{array}\right] .
\end{aligned}
$$

As shown in Appendix A, the state space realization of the model reduction error $K(s)=G(s)-G_{r}(s)$ is given by 


$$
\begin{aligned}
{\left[\begin{array}{l}
\dot{z}_{1} \\
\dot{z}_{2}
\end{array}\right] } & =\left[\begin{array}{ll}
\hat{F}_{11} & 0 \\
\hat{F}_{21} & \hat{F}_{22}
\end{array}\right]\left[\begin{array}{l}
z_{1} \\
z_{2}
\end{array}\right]+\left[\begin{array}{c}
\Gamma_{1} \\
-E_{22}
\end{array}\right] u \\
y & =\left[\begin{array}{ll}
\hat{H}_{1} & \hat{H}_{2}
\end{array}\right]\left[\begin{array}{l}
z_{1} \\
z_{2}
\end{array}\right] .
\end{aligned}
$$

where $-E_{2}^{T} Q^{T}$ is partitioned conformably as $\left[\Gamma_{1}^{T},-E_{22}^{T}\right]$.

We now seek to compute an upper bound on the $H_{2}$ norm of the model error $K(s)$ when $\alpha=0$. To simplify the notation we shall denote $\left[\Gamma_{1}^{T},-E_{22}{ }^{T}\right]$ by $E_{C}{ }^{T}$. Using eqn. (5.35) from [11] to compute the $\mathrm{H}_{2}$ norm of $G(s)$ and $K(s)$ we have:

$$
\begin{aligned}
\|G\|_{2}^{2} & =\operatorname{trace}\left(\Gamma^{T} W_{o b s} \Gamma\right) \\
\|K\|_{2}^{2} & =\operatorname{trace}\left(E_{C}^{T} W_{o b s} E_{C}\right)
\end{aligned}
$$

where $W_{o b s}$ is the observability grammian of both $G(s)$ and $K(s)$. Let $W_{o b s}=L L^{T}$ be the cholesky factors for $W_{o b s}$, a positive definite symmetric matrix with eigenvalues we will call $\sigma_{1}^{2}, \ldots \sigma_{p}^{2}$. Then

$$
\operatorname{trace}\left(W_{o b s}\right)=\sigma_{1}^{2}+\cdots+\sigma_{p}^{2}=\|L\|_{F}^{2} .
$$

In general, $\operatorname{trace}\left(M^{T} M\right) \equiv\|M\|_{F}^{2}$.

Then we have

$$
\operatorname{trace}\left(E_{C}^{T} W_{o b s} E_{C}\right)=\operatorname{trace}\left(E_{C}^{T} L L^{T} E_{C}\right)=\left\|L^{T} E_{C}\right\|_{F}^{2} \leq\|L\|_{F}^{2}\left\|E_{C}\right\|_{F}^{2}=\operatorname{trace}\left(W_{o b s}\right) \cdot\left\|E_{C}\right\|_{F}^{2} .
$$

Now $\left\|E_{C}\right\|_{F}$ is small since $E_{C}$ is just the perturbation we are committing to arrive at the uncontrollable system to truncate.

Here is an "analysis" for the term trace $\left(W_{o b s}\right)$. Roughly, this term measures the energy that can be retrieved in the output from the system states, and $\|G\|_{2}^{2}$ measures the energy that can be retrieved in the output from a unit impulse input. Hence the ratio: 


$$
\rho_{\text {input }}=\frac{\sqrt{\operatorname{trace}\left(W_{o b s}\right)}}{\|G\|}
$$

roughly measures the input transmission ratio of energy. We may similarly define the output transmission ratio of energy as

$$
\rho_{\text {output }}=\frac{\sqrt{\operatorname{trace}\left(W_{\text {contr }}\right)}}{\|G\|} .
$$

These ratios are properties inherent to a given state-space realization, regardless of whatever model reduction method is used.

Using this ratio, we may bound the norm of the error transfer function $K(s)$ using eqn. (64):

$$
\|K\| \leq \sqrt{\operatorname{trace}\left(W_{\text {obs }}\right)} \cdot\left\|E_{C}\right\|_{F}=\rho_{\text {input }} \cdot\|G\| \cdot\left\|E_{C}\right\|_{F} .
$$

We have computed the reduced order system by applying a controllability perturbation to the inputs. We could also apply an observability perturbation to the outputs, obtaining a reduced order model $\hat{G}_{r}(s)$ and error transfer function $K_{o b s}(s)=G(s)-\hat{G}_{r}(s)$. Working through the same development, we could obtain the bound

$$
\left\|K_{\text {obs }}\right\| \leq \sqrt{\operatorname{trace}\left(W_{\text {contr }}\right)} \cdot\left\|E_{O}\right\|_{F}=\rho_{\text {output }} \cdot\|G\| \cdot\left\|E_{O}\right\|_{F},
$$

where $E_{O}$ is the perturbation to the output matrix $H$.

Note that $\rho_{\text {input }}>\rho_{\text {output }}$ is equivalent to trace $\left(W_{\text {contr }}\right)<\operatorname{trace}\left(W_{\text {obs }}\right)$. As discussed in subsection 3.5, the trace of the controllability and observability grammians give a criteria for deciding whether to effect model reduction by input or output perturbation. Because of the equivalence relationship between the ratios and the trace of the grammians, the criteria for deciding which method to choose can be stated as follows: if $\rho_{\text {input }}>\rho_{\text {output }}$ choose the input perturbation method otherwise choose the output perturbation method.

In the case of the first example in section 6, the computed values for the ratios are 


$$
\rho_{\text {input }}=21.0970 \quad \text { and } \quad \rho_{\text {output }}=0.0211 ;
$$

and for the second example, these ratios can be computed for the strictly proper part of model as

$$
\rho_{\text {input }}=3.6417 \text { and } \quad \rho_{\text {output }}=0.2821 \text {. }
$$

These numbers suggest that the controllability method should be used in both cases to implement the model reduction.

When $\alpha \neq 0$ then in general the eigenvalues of the full order model are different from the eigenvalues of the reduced order model and we do not have any simple way of expressing the model error dynamics. However we can augment the states $x$ of the full order model with the states $z_{2}$ of the reduced order model to form a composite system with states $\left(x, z_{2}\right)$. From equations (2) and (24) we form the state space representation for the model reduction error $K(s)$ as

$$
\begin{aligned}
{\left[\begin{array}{l}
\dot{x} \\
\dot{z}_{2}
\end{array}\right] } & =\left[\begin{array}{cc}
F & 0 \\
0 & \tilde{F}_{22}
\end{array}\right]\left[\begin{array}{l}
x \\
z_{2}
\end{array}\right]+\left[\begin{array}{c}
G \\
\tilde{G}_{2}
\end{array}\right] u \\
y_{e} & =\left[\begin{array}{ll}
H & -\tilde{H}_{2}
\end{array}\right]\left[\begin{array}{l}
x \\
z_{2}
\end{array}\right] .
\end{aligned}
$$

For both $\alpha=0$ and $\alpha \neq 0$, the state space expression for the model reduction error is in terms of the full order model, the perturbation $E_{i}$ and the orthonormal matrix $Q$. The sup-norm of the model reduction error can be computed using an iteration method such as [12]. 


\section{Numerical Results}

We applied the matrix pencil reduction scheme described in section 3 using the controllability condition. The " $D$ " and " $C$ " matrices were chosen as outlined in section 5 with $\alpha=1$. The system matrices for both examples are listed in Appendix B. In both cases the reduced order model was obtained by implementing the method outlined in subsection 3.2.

\section{Example 1}

The first example is the "JPL 8-state model" taken from page 32 of [13], which is a model of SISO large flexible structure. The poles and zeros are listed in Table 1. Figure 1.1 is a Bode magnitude and phase plots, and Figure 1.2 is the pole-zero plot of the original 8 states system.

\begin{tabular}{|c|c|}
\hline \multicolumn{1}{|c|}{$i$} & \multicolumn{1}{c|}{$\lambda_{i}[F]$} \\
\hline 1,2 & $-0.2119 \pm j 10.5931$ \\
\hline 3,4 & $-0.0736 \pm j 0.6714$ \\
\hline 5,6 & $-0.3370 \pm j 16.8473$ \\
\hline 7,8 & $-0.0528 \pm j 3.9405$ \\
\hline
\end{tabular}

Table 1 Poles and Zeros of full order model listed in no particular order.

Table 2 shows the norm of the perturbations and the corresponding eigenvalues of $[A, C]$.

\begin{tabular}{||l|l|l||}
\hline \multicolumn{1}{|c|}{$\boldsymbol{i}$} & \multicolumn{1}{c|}{$\left\|\boldsymbol{E}_{\boldsymbol{i}}\right\|$} & \multicolumn{1}{c|}{$\lambda_{i}[\boldsymbol{A}, \boldsymbol{C}]$} \\
\hline 1,2 & 0.0047 & $-0.2119 \pm j 10.5931$ \\
\hline 3,4 & 0.0160 & $-0.3370 \pm j 16.8473$ \\
\hline 5,6 & 0.0492 & $-0.0529 \pm j 3.9405$ \\
\hline 7,8 & 0.0608 & $-0.0772 \pm j 0.6771$ \\
\hline 9 & 1.0534 & 0.9948 \\
\hline
\end{tabular}

Table $2 \quad\left\|E_{i}\right\|$ and the corresponding eigenvalues of $[A, C]$ sorted by $\left\|E_{i}\right\|$.

Note the gap in the norm of the perturbations corresponding to the $8^{\text {th }}$ and $9^{\text {th }}$ eigenvalues in 
Table 2. We thus identify the eigenvalue $\lambda_{9}$ of $[A, C]$ as a spurious eigenvalue of $F$.

The complex eigenpair $\left(\lambda_{1}, v_{1}\right)$ was used to construct the perturbation matrix $E_{i}$ for the reduced order 6 model, whereas the eigenpairs $\left\{\left(\lambda_{1}, v_{1}\right),\left(\lambda_{3}, v_{3}\right)\right\}$ were used to obtain a reduced order 4 model. Figure 1.3 is the Bode magnitude and phase plots of the full order and the reduced order 6 model; Figure 1.4 shows same plots for reduced order 4 model.

We next compared the performance of the matrix pencil reduction scheme with 1) truncation by balanced realization [7], and 2) optimal Hankel norm reduction [9] methods. The balanced realization reduced order model was obtained by using the functions "balreal" in [15] and "strunc" in [16]; and the optimal Hankel norm reduced order model was obtained by using the functions "sysbal" and "hankmr" from [16]. Figure 1.5 shows the Bode plots of the original system and the reduced order 6 models obtained from optimal Hankel norm and balanced realization methods. Figure 1.6 is the corresponding error magnitude plots for all three methods; the error is defined as $G(j \omega)-G_{r}(j \omega)$ where $G(j \omega)$ is the frequency response of the original system and $G_{r}(j \omega)$ is the frequency response of the reduced order model. From Figures 1.3 and 1.5 , we see that both the matrix pencil and the balanced realization methods yield a reduced order 6 model whose Bode plots are identical to those of the original system except around a small frequency band at 10rad/s. The reduced order 6 model from the optimal Hankel norm method also approximates Bode plots of the original system well except around the small frequency band at $10 \mathrm{rad} / \mathrm{s}$ and at high frequencies.

Figure 1.7 shows the Bode plots of the original system and the reduced order 4 models using the two other methods, and Figure 1.8 is the corresponding error magnitude plots for all three methods. In this case the optimal Hankel norm approach gives the worst fit. Both the matrix pencil and balanced realization methods fit the Bode plots of the original system well at low and intermediate frequencies.

The Bode phase plots show that the phase error over the entire frequency range is least for the models obtained from the matrix pencil approach. All three methods give approximately the same peak-norm of the error for both reduced order models of 6 and 4 states. 


\section{Example 2}

The second example is a MIMO model of the "CSI evolutionary" structure [14]; the model which was used in this paper was furnished by Prof. Gary Balas. The original system (i.e., the full order model) consists of 26 states, 8 inputs and 10 outputs. Figure 2.1 is a plot of the maximum and minimum singular values of the system transfer function matrix. Figure 2.2 is a plot of the eigenvalues of $F$ which are also listed in Table 3; the original system has no finite transmission zero.

\begin{tabular}{||l|l||}
\hline \multicolumn{1}{|c|}{$i$} & \multicolumn{1}{c|}{$\lambda_{i}[F]$} \\
\hline 1,2 & $-0.0351 \pm j 0.9264$ \\
\hline 3,4 & $-0.0009 \pm j 0.9393$ \\
\hline 5,6 & $-0.0490 \pm j 0.9747$ \\
\hline 7,8 & $-0.0236 \pm j 4.5992$ \\
\hline 9,10 & $-0.0195 \pm j 4.7232$ \\
\hline 11,12 & $-0.0166 \pm j 5.5857$ \\
\hline 13,14 & $-0.0238 \pm j 9.3098$ \\
\hline 15,16 & $-0.0141 \pm j 14.1052$ \\
\hline 17,18 & $-0.0967 \pm j 15.1058$ \\
\hline 19,20 & $-0.0159 \pm j 15.8949$ \\
\hline 21,22 & $-0.1001 \pm j 16.2444$ \\
\hline 23,24 & $-0.2588 \pm j 18.2781$ \\
\hline 25,26 & $-0.0215 \pm j 21.4750$ \\
\hline
\end{tabular}

Table 3 Eigenvalues of $F$ listed in no particular order.

Table 4 shows the norm of the perturbations and the corresponding eigenvalues of $[A, C]$. Once again, note the gap in the norm of the perturbations corresponding to the $26^{\text {th }}$ and $27^{\text {th }}$ eigenvalues in the table. We thus identify all the eigenvalues whose $\left\|E_{i}\right\|$ is greater than or equal to 1.4207 as spurious eigenvalues. 


\begin{tabular}{||l|l|l||}
\hline \multicolumn{1}{|c|}{$\boldsymbol{i}$} & \multicolumn{1}{|c|}{$\left\|\boldsymbol{E}_{\boldsymbol{i}}\right\|$} & \multicolumn{1}{|c|}{$\lambda_{i}[\boldsymbol{A}, \boldsymbol{C}]$} \\
\hline 1,2 & 0.0103 & $-0.0215 \pm j 21.4750$ \\
\hline 3,4 & 0.0759 & $-0.0159 \pm j 15.8949$ \\
\hline 5,6 & 0.1200 & $-0.0142 \pm j 14.1052$ \\
\hline 7,8 & 0.1629 & $-0.2589 \pm j 18.2781$ \\
\hline 9,10 & 0.1715 & $-0.0207 \pm j 4.7235$ \\
\hline 11,12 & 0.2712 & $-0.0269 \pm j 4.6000$ \\
\hline 13,14 & 0.2775 & $-0.0247 \pm j 9.3099$ \\
\hline 15,16 & 0.2882 & $-0.0191 \pm j 5.5861$ \\
\hline 17,18 & 0.2968 & $-0.1004 \pm j 16.2444$ \\
\hline 19,20 & 0.3117 & $-0.0809 \pm j 0.9718$ \\
\hline 21,22 & 0.3575 & $-0.0972 \pm j 15.1058$ \\
\hline 23,24 & 0.3971 & $-0.0751 \pm j 1.0109$ \\
\hline 25,26 & 0.4860 & $-0.1369 \pm j 1.0555$ \\
\hline 27 & 1.4207 & 0.9980 \\
\hline 28 & 1.4500 & 1.0021 \\
\hline 29 & 1.4567 & 1.0034 \\
\hline 30 & 1.4881 & 1.0109 \\
\hline 31 & 2.2181 & 1.0006 \\
\hline 32 & 2.2947 & 1.0010 \\
\hline 33 & 2.5031 & 1.0039 \\
\hline 34 & 4.1977 & 1.0000 \\
\hline & & \\
\hline 34
\end{tabular}

Table $4\left\|E_{i}\right\|$ and the corresponding eigenvalues of $[A, C]$ sorted by $\left\|E_{i}\right\|$. 
The eigenpair $\left(\lambda_{1}, v_{1}\right)$ was used to generate $E_{i}$ for reduced order model of 24 states, and the pairs $\left\{\left(\lambda_{1}, v_{1}\right),\left(\lambda_{3}, v_{3}\right)\right\}$ were used for reduced order model of 22 states. For reduced order models of order 24 and 22 there is no noticeable difference between singular values plot of the original and the reduced order models. The eigenpairs $\left\{\left(\lambda_{1}, v_{1}\right),\left(\lambda_{3}, v_{3}\right),\left(\lambda_{5}, v_{5}\right)\right\}$ were used to generate $E_{i}$ for reduced order 20 model, and for reduced order 16 model the eigenpairs $\left\{\left(\lambda_{1}, v_{1}\right),\left(\lambda_{3}, v_{3}\right),\left(\lambda_{5}, v_{5}\right)\right.$, $\left.\left(\lambda_{7}, v_{7}\right),\left(\lambda_{9}, v_{9}\right)\right\}$ were used. Figure 2.3 is a plot of the maximum and minimum singular values of the reduced order model of 20 states; Figure 2.4 shows same plot for reduced order model of 16 states. As can be seen from Figure 2.3, for the reduced order 20 model, the singular values plots are almost identical with those of the full order model of 26 states.

As is well known, the SISO notion of phase is not easily generalized for the MIMO system. However, in some applications, phase information of the individual transfer functions are important. We thus decided to verify how closely the Bode plots of the individual transfer functions of the reduced order models follow that of the full order model. Since the MIMO transfer function matrix has $8 \times 10$ elements (and we do not want to generate that many plots) we picked the transfer function between the first output and the first input, i.e., the $(1,1)$ entry for this purpose. Figure 2.5 is the Bode magnitude and phase plots of the $(1,1)$ transfer function for reduced order 20 model; and Figure 2.6 shows Bode plots of the $(1,1)$ transfer function for reduced order 16 model. In both cases, the phase plots of the reduced order models closely match that of the full order model.

Once again, we compared the performance of the matrix pencil reduction scheme with Hankel norm reduction and balanced realization reduction methods. Figure 2.7 is a plot of the maximum and minimum singular values versus frequency of the reduced order models of 20 states using the optimal Hankel norm and balanced realization methods. Figure 2.8 is the plot of the maximum singular values of the resulting error for the three different methods. By comparing Figures 2.3, 2.7 and 2.8, we see that the optimal Hankel norm method gives the worst fit of the singular values however, it also has the smallest peak-norm of the error. The matrix pencil method, on the other hand gives the best fit however, it has the largest peak-norm of the error. The model from balanced realization gives a good fit at intermediate and high frequencies but a very poor fit at low frequencies. Figure 2.9 shows the Bode magnitude and phase plots for the reduced order 20 models of the $(1,1)$ transfer function using the two other methods. By comparing Figures 2.5 and 2.9 we see that the matrix pencil approach approximates both the phase and magnitude of the full order model much better than the other two methods. 
Figure 2.10 is a plot of the maximum and minimum singular values versus frequency of the reduced order 16 models obtained from the other two methods. Figure 2.11 is the plot of the maximum singular values of the resulting error for the three different methods. By comparing Figures 2.4, 2.10 and 2.11 we see that the optimal Hankel norm method gives the worst fit and the highest peak-norm of the error. The model from balanced realization has the smallest peaknorm of the error, however, it gives a very poor fit of the singular values in the low frequency range. The model from matrix pencil method has a higher peak-norm of error than the model from balanced realization, nevertheless it gives the best fit. Figure 2.12 shows the Bode magnitude and phase plots for the reduced order models of 16 states of the $(1,1)$ transfer function obtained from the other two methods. Once again, from Figures 2.6 and 2.12 we see that the matrix pencil approach approximates both the phase and magnitude much better than the other two methods.

\section{Conclusion}

In this paper we have presented a novel way of model reduction based on matrix pencil theory. We have given some initial results on the stability of the reduced order model in terms of a perturbation applied to the system matrix $F$. However, for the special case of $\alpha=0$, the reduced order model is always stable so long as the full order model is. We have also derived an upper bound on the $\mathrm{H}_{2}$ norm of the model reduction error when $\alpha=0$. The computational cost of the matrix pencil method is comparable to other methods which require balanced realization. Some of the advantages of the matrix pencil method over the balanced realization and optimal Hankel norm methods are: 1) there is no need to transform the full order model into balanced realization, and 2) the matrix pencil method uses only orthonormal transformations.

We have given two detailed examples to illustrate features of the method. In both cases, the matrix pencil yields models that are much better approximations than those from the optimal Hankel norm method. The models from the matrix pencil method tend to follow the phase of the full order model better than models from the balanced realization method, and are otherwise comparable. 


\section{REFERENCES}

[1] D. Boley, Estimating the Sensitivity of the Algebraic Structure of Pencils with Simple Eigenvalue Estimates, SIAM J. Matrix Anal. Appl., Vol. 11, No. 4, Oct. 1990, p.p 632643.

[2] D. Boley, Computing Rank-Deficiency of Rectangular Matrix Pencils, Systems Control Lett., 9 (1986), pp. 207-214.

[3] P. Van Dooren, The Computation of Kronecker's Canonical Form of a Singular Pencil, Linear Algebra Appl., 27 (1979) pp. 103-141.

[4] T. Kailath, Linear Systems, Prentice-Hall, Englewood Cliffs, NJ, 1980.

[5] F. R. Gantmakher, Theory of Matrices, Vols. 1 and 2, Chelsea, New York, 1959.

[6] G. H. Golub and C. Van Loan, Matrix Computations, The John Hopkins University Press, Baltimore, MD, 1983.

[7] B. C. Moore, "Principal component analysis in linear systems: controllability, observability, and model reduction," IEEE Trans. Auto. Control, Vol. AC-26, No. $1,1981$.

[8] D. Enns, "Model reduction for control system design," Ph.D. dissertation, Dept. of Aeronautics and Astronautics, Stanford University, June 1984.

[9] K. Glover, "All optimal Hankel-norm approximations of linear multivariable systems and their $L_{\infty}$-error bounds," IJC, 1984, Vol 39, No. 6, 1115-1193.

[10] G. O. Lathan and B.D.O. Anderson, "Frequency-Weighted optimal Hankel-norm approximation of stable transfer functions," Systems \& Control Letters 5 (1985) 229-236.

[11] S. P. Boyd and C. M. Barratt, Linear Controller Design: Limits of Performance. PrenticeHall, 1991. 
[12] S. Boyd, V. Balakrishnan, and P. Kabamba, " A Bisection Method for Computing the $H_{\infty}$ Norm of a Transfer Matrix and Related Problems," Math Control Signals and Systems, 2(3): 207-219, 1989.

[13] S.A. Buddie, T.T. Georgiou, U. Ozguner, and M.C. Smith, Flexible Structure Experiments at JPL and WPAFB: $H_{\infty}$ Controller Designs, CUED/F-INFENG/TR 99, University of Cambridge, Dept. of Engineering, Cambridge, U.K., May 1992.

[14] K. B. Kim, P.G. Maghami, and S. M. Joshi, Comparison of Controller Designs for an Experimental Flexible Structure, IEEE Control Systems Magazine, Vol 12, No 3, June 1992, pp. 108-117.

[15] A. Grace, A.J. Laub, J.N. Little, J. N. Little, and C. Thompson, Control System Toolbox for use with MATLAB, The MATHWORKS Inc., MA., USA, Nov. 1990.

[16] G.J. Balas, J.D. Doyle, K. Glover, A.K. Packard, and R. Smith, $\mu$-Analysis and Synthesis Toolbox, The MATHWORKS Inc., MA. and MUSYN Inc., Minneapolis, MN, USA, April 1991. 


\section{Appendix A}

In this section we derive state space expressions for the model reduction error when $\alpha=0$. We shall assume that the reduced order model was obtained using the controllability condition. The transfer function matrix of the state space model of eqn. (59) is given by

$$
G(s)=\left[\begin{array}{ll}
\hat{H}_{1} & \hat{H}_{2}
\end{array}\right]\left[\begin{array}{cc}
s I-\hat{F}_{11} & 0 \\
-\hat{F}_{21} & s I-\hat{F}_{22}
\end{array}\right]^{-1}\left[\begin{array}{c}
\Gamma_{1} \\
\Gamma_{2}
\end{array}\right]
$$

let

$$
\Delta=\left(s I-\hat{F}_{11}\right)^{-1}, \quad \text { and } \quad \phi=\left(s I-\hat{F}_{22}\right)^{-1}
$$

then application of the matrix inversion lemma [4] to (A.1) yields

$$
\begin{aligned}
G(s) & =\left[\begin{array}{ll}
\hat{H}_{1} & \hat{H}_{2}
\end{array}\right]\left[\begin{array}{cc}
\Delta & 0 \\
-\phi\left(-\hat{F}_{21}\right) \Delta & \phi
\end{array}\right]\left[\begin{array}{l}
\Gamma_{1} \\
\Gamma_{2}
\end{array}\right] \\
& =\hat{H}_{1} \Delta \Gamma_{1}+\hat{H}_{2} \phi \hat{F}_{21} \Delta \Gamma_{1}+\hat{H}_{2} \phi \Gamma_{2} .
\end{aligned}
$$

Now the state space representation of the reduced order model is

$$
\begin{aligned}
\dot{z}_{2} & =\tilde{F}_{22} z_{2}+\tilde{G}_{2} u \\
y & =\tilde{H}_{2} z_{2}
\end{aligned}
$$

where

$$
\tilde{F}_{22}, \tilde{G}_{2}, \text { and } \tilde{H}_{2}
$$

are defined in eqn. (24) with $E_{1}=0$. Note that

$$
\tilde{F}=\hat{F}, \tilde{H}=\hat{H}, \quad \text { which implies that } \tilde{F}_{22}=\hat{F}_{22} \text {, and } \tilde{H}_{2}=\hat{H}_{2} \text {. }
$$

Denote the transfer matrix of the reduced order model by $G_{r}(s)$, i.e., 


$$
\begin{aligned}
G_{r}(s) & =\tilde{H}_{2}\left(s I-\tilde{F}_{22}\right) \tilde{G}_{2} \\
& =\tilde{H}_{2} \phi \tilde{G}_{2}
\end{aligned}
$$

then the model reduction error $K(s)$ is given by

$$
K(s) \equiv G(s)-G_{r}(s)=\hat{H}_{1} \Delta \Gamma_{1}+\hat{H}_{2} \phi \hat{F}_{21} \Delta \Gamma_{1}+\hat{H}_{2} \phi\left(\Gamma_{2}-\tilde{G}_{2}\right) .
$$

Now

$$
\Gamma-\tilde{G}=Q\left(G-\left(G+E_{2}\right)\right)=-Q E_{2} .
$$

If we write $Q E_{2}$ as

$$
Q E_{2}=\left[\begin{array}{c}
E_{21} \\
E_{22}
\end{array}\right],
$$

where $E_{21}$ and $E_{22}$ have the dimensions of $\Gamma_{1}$ and $\Gamma_{2}$ respectively, then

$$
K(s)=\hat{H}_{1} \Delta \Gamma_{1}+\hat{H}_{2} \phi \hat{F}_{21} \Delta \Gamma_{1}-\hat{H}_{2} \phi E_{22} .
$$

The RHS of (A.11) can be written as

$$
\left[\begin{array}{ll}
\hat{H}_{1} & \hat{H}_{2}
\end{array}\right]\left[\begin{array}{cc}
\Delta & 0 \\
\phi \hat{F}_{21} \Delta & \phi
\end{array}\right]\left[\begin{array}{c}
\Gamma_{1} \\
-E_{22}
\end{array}\right]
$$

which in turn has the following state space realization

$$
\begin{aligned}
{\left[\begin{array}{l}
\dot{z}_{1} \\
\dot{z}_{2}
\end{array}\right] } & =\left[\begin{array}{ll}
\hat{F}_{11} & 0 \\
\hat{F}_{21} & \hat{F}_{22}
\end{array}\right]\left[\begin{array}{l}
z_{1} \\
z_{2}
\end{array}\right]+\left[\begin{array}{c}
\Gamma_{1} \\
-E_{22}
\end{array}\right] u \\
y & =\left[\begin{array}{ll}
\hat{H}_{1} & \hat{H}_{2}
\end{array}\right]\left[\begin{array}{l}
z_{1} \\
z_{2}
\end{array}\right] .
\end{aligned}
$$




\section{Appendix B}

System matrices $(F, G, H, J)$ for examples 1 and 2 in section 7 . The transfer function matrix is $J+H(s I-F)^{-1} G$.

1. Example 1: Data taken from page 32 of [13].

» printsys $(\mathrm{F}, \mathrm{G}, \mathrm{H}, \mathrm{J})$

$F=$

$\begin{array}{rrrrrrrrr} & \mathrm{x} 1 & \mathrm{x} 2 & \mathrm{x} 3 & \mathrm{x} 4 & \mathrm{x} 5 & \mathrm{x} 6 & \mathrm{x} 7 & \mathrm{x} 8 \\ \mathrm{x} 1 & 0 & 0 & 0 & 0 & 1.0000 & 0 & 0 & 0 \\ \mathrm{x} 2 & 0 & 0 & 0 & 0 & 0 & 1.0000 & 0 & 0 \\ \mathrm{x} 3 & 0 & 0 & 0 & 0 & 0 & 0 & 1.0000 & 0 \\ \mathrm{x} 4 & 0 & 0 & 0 & 0 & 0 & 0 & 0 & 1.0000 \\ \mathrm{x} 5 & -112.2586 & 0 & 0 & 0 & -0.4238 & 0 & 0 & 0 \\ \mathrm{x} 6 & 0 & -283.9449 & 0 & 0 & 0 & -0.6740 & 0 & 0 \\ \mathrm{x} 7 & 0 & 0 & -0.4562 & 0 & 0 & 0 & -0.1472 & 0 \\ \mathrm{x} 8 & 0 & 0 & 0 & -15.5305 & 0 & 0 & 0 & -0.1056\end{array}$

$G=$

u1

$\begin{array}{lr}\mathrm{x} 1 & 0 \\ \mathrm{x} 2 & 0 \\ \mathrm{x} 3 & 0 \\ \mathrm{x} 4 & 0 \\ \mathrm{x} 5 & -0.0495 \\ \mathrm{x} 6 & 0.2706 \\ \mathrm{x} 7 & -0.0730 \\ \mathrm{x} 8 & -0.1998\end{array}$

$H=$

\begin{tabular}{|c|c|c|c|c|c|c|}
\hline $\mathrm{x} 1$ & $\mathrm{x} 2$ & x3 & $\mathrm{x} 4$ & $x 5$ & $x 6$ & $\times 7$ \\
\hline-49.4740 & 270.5736 & -72.9940 & -199.8225 & 0 & 0 & 0 \\
\hline
\end{tabular}

$J=$

$\begin{array}{rr}\mathrm{u} 1 \\ \mathrm{y} 1 & 0\end{array}$ 
Example 2: Data supplied by Prof. Gary Balas.

» printsys $(\mathrm{F}, \mathrm{G}, \mathrm{H}, \mathrm{J})$

$\mathrm{F}=$

\begin{tabular}{|c|c|c|c|c|c|c|c|c|c|c|c|c|c|}
\hline & $\mathrm{x} 1$ & $\mathrm{x} 2$ & $\mathrm{x} 3$ & $\mathrm{x} 4$ & $x 5$ & $x 6$ & $x 7$ & $\mathrm{x} 8$ & $x 9$ & $\mathrm{x} 10$ & $\mathrm{x} 11$ & $\mathrm{x} 12$ & $\mathrm{x} 13$ \\
\hline x1 & -0.03512 & -0.92641 & 0 & 0 & 0 & 0 & 0 & 0 & 0 & 0 & 0 & 0 & 0 \\
\hline$x 2$ & 0.92641 & -0.03512 & 0 & 0 & 0 & 0 & 0 & 0 & 0 & 0 & 0 & 0 & 0 \\
\hline $\mathrm{x} 4$ & 0 & 0 & 0.93933 & -0.00094 & 0 & 0 & 0 & 0 & 0 & 0 & 0 & 0 & 0 \\
\hline$x 5$ & 0 & 0 & 0 & 0 & -0.04896 & -0.97466 & 0 & 0 & 0 & 0 & 0 & 0 & 0 \\
\hline$x 6$ & 0 & 0 & 0 & 0 & 0.97466 & -0.04896 & 0 & 0 & 0 & 0 & 0 & 0 & 0 \\
\hline$x 7$ & 0 & 0 & 0 & 0 & 0 & 0 & -0.02364 & -4.59925 & 0 & 0 & 0 & 0 & 0 \\
\hline$x 8$ & 0 & 0 & 0 & 0 & 0 & 0 & 4.59925 & -0.02364 & 0 & 0 & 0 & 0 & 0 \\
\hline x9 & 0 & 0 & 0 & 0 & 0 & 0 & 0 & 0 & -0.01946 & -4.72321 & 0 & 0 & 0 \\
\hline $\mathrm{x} 10$ & 0 & 0 & 0 & 0 & 0 & 0 & 0 & 0 & 4.72321 & -0.01946 & 0 & 0 & 0 \\
\hline x11 & 0 & 0 & 0 & 0 & 0 & 0 & 0 & 0 & 0 & 0 & -0.01659 & -5.58565 & 0 \\
\hline $\mathrm{x} 12$ & 0 & 0 & 0 & 0 & 0 & 0 & 0 & 0 & 0 & 0 & 5.58565 & -0.01659 & $9 \quad 0$ \\
\hline $\mathrm{x} 13$ & 0 & 0 & 0 & 0 & 0 & 0 & 0 & 0 & 0 & 0 & 0 & 0 & -0.02383 \\
\hline x14 & 0 & 0 & 0 & 0 & 0 & 0 & 0 & 0 & 0 & 0 & 0 & 0 & 9.30982 \\
\hline x15 & 0 & 0 & 0 & 0 & 0 & 0 & 0 & 0 & 0 & 0 & 0 & 0 & 0 \\
\hline $\mathrm{x} 17$ & 0 & 0 & 0 & 0 & 0 & 0 & 0 & 0 & 0 & 0 & 0 & 0 & 0 \\
\hline x18 & 0 & 0 & 0 & 0 & 0 & 0 & 0 & 0 & 0 & 0 & 0 & 0 & 0 \\
\hline x19 & 0 & 0 & 0 & 0 & 0 & 0 & 0 & 0 & 0 & 0 & 0 & 0 & 0 \\
\hline $\mathrm{x} 20$ & 0 & 0 & 0 & 0 & 0 & 0 & 0 & 0 & 0 & 0 & 0 & 0 & 0 \\
\hline $\mathrm{x} 21$ & 0 & 0 & 0 & 0 & 0 & 0 & 0 & 0 & 0 & 0 & 0 & 0 & 0 \\
\hline$x 22$ & 0 & 0 & 0 & 0 & 0 & 0 & 0 & 0 & 0 & 0 & 0 & 0 & 0 \\
\hline$x 23$ & 0 & 0 & 0 & 0 & 0 & 0 & 0 & 0 & 0 & 0 & 0 & 0 & 0 \\
\hline $\mathrm{x} 24$ & 0 & 0 & 0 & 0 & 0 & 0 & 0 & 0 & 0 & 0 & 0 & 0 & 0 \\
\hline $\mathrm{x} 25$ & 0 & 0 & 0 & 0 & 0 & 0 & 0 & 0 & 0 & 0 & 0 & 0 & 0 \\
\hline x 26 & 0 & 0 & 0 & 0 & 0 & 0 & 0 & 0 & 0 & 0 & 0 & 0 & 0 \\
\hline
\end{tabular}


( $\mathrm{F}$ continued)

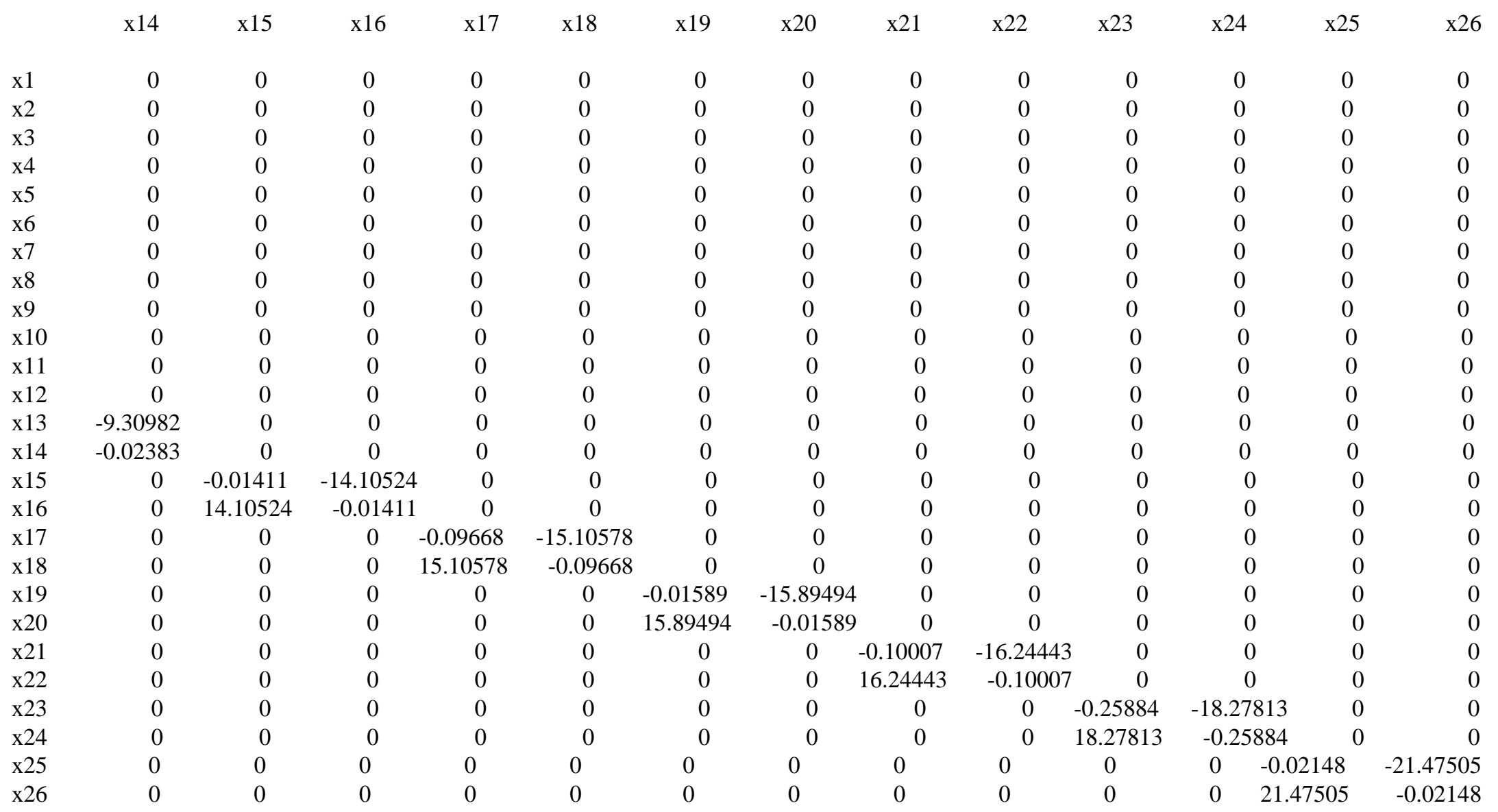




\begin{tabular}{|c|c|c|c|c|c|c|c|c|}
\hline & $\mathrm{u} 1$ & u2 & u3 & $\mathrm{u} 4$ & u5 & u6 & u7 & $\mathrm{u} 8$ \\
\hline $\mathrm{x} 1$ & -0.03538 & -0.00038 & -0.00595 & 0.00013 & 0.22276 & 0.02065 & 0.22244 & -0.02000 \\
\hline $\mathrm{x} 2$ & 0.03302 & 0.00036 & 0.00555 & -0.00013 & -0.20790 & -0.01928 & -0.20760 & 0.01866 \\
\hline$x 3$ & 0.24290 & $-7.43881 \mathrm{e}-05$ & 0.21926 & $-5.12698 \mathrm{e}-05$ & 0.00592 & 0.18554 & 0.00591 & 0.20004 \\
\hline$x 4$ & -0.24409 & $7.47521 \mathrm{e}-05$ & -0.22033 & $5.15206 \mathrm{e}-05$ & -0.00595 & -0.18645 & -0.00594 & -0.20102 \\
\hline x5 & -0.31529 & $4.42235 \mathrm{e}-05$ & 0.00463 & $-9.80589 e-06$ & -0.01924 & 0.29025 & -0.01921 & -0.15788 \\
\hline x6 & 0.28718 & $-4.02810 \mathrm{e}-05$ & -0.00422 & $8.93170 \mathrm{e}-06$ & 0.01752 & -0.26438 & 0.01750 & 0.14381 \\
\hline $\mathrm{x} 7$ & $3.24533 \mathrm{e}-05$ & 0.25788 & $1.81223 \mathrm{e}-05$ & 0.04691 & 0.01627 & $-5.27259 \mathrm{e}-05$ & 0.05441 & $-9.96934 e-05$ \\
\hline $\mathrm{x} 8$ & $-3.32222 \mathrm{e}-05$ & -0.26399 & $-1.85516 \mathrm{e}-05$ & -0.04802 & -0.01665 & $5.39751 \mathrm{e}-05$ & -0.05570 & 0.00010 \\
\hline x9 & $4.29716 \mathrm{e}-06$ & -0.08797 & $2.24446 \mathrm{e}-05$ & -0.14337 & 0.00410 & $-4.30098 \mathrm{e}-05$ & 0.01508 & -0.00012 \\
\hline $\mathrm{x} 10$ & $-4.41196 e-06$ & 0.09032 & $-2.30442 \mathrm{e}-05$ & 0.14720 & -0.00421 & $4.41588 \mathrm{e}-05$ & -0.01548 & 0.00012 \\
\hline $\mathrm{x} 11$ & 0.01522 & -0.00065 & 0.04909 & -0.00047 & -0.00013 & -0.05881 & -0.00024 & -0.27181 \\
\hline $\mathrm{x} 12$ & -0.01576 & 0.00068 & -0.05084 & 0.00048 & 0.00014 & 0.06090 & 0.00025 & 0.28149 \\
\hline$x 13$ & -0.10574 & 0.00073 & 0.03091 & 0.00011 & -0.00026 & -0.05774 & 0.00061 & 0.23805 \\
\hline x14 & 0.11263 & -0.00078 & -0.03292 & -0.00011 & 0.00027 & 0.06151 & -0.00065 & -0.25355 \\
\hline $\mathrm{x} 15$ & 0.07063 & 0.00197 & -0.05541 & -0.00170 & -0.00106 & 0.06223 & 0.00188 & -0.03160 \\
\hline$x 16$ & -0.07817 & -0.00218 & 0.06132 & 0.00188 & 0.00118 & -0.06887 & -0.00208 & 0.03498 \\
\hline$x 17$ & 0.00235 & 0.21299 & -0.00205 & -0.18416 & -0.07232 & 0.00038 & 0.17557 & 0.00095 \\
\hline $\mathrm{x} 18$ & -0.00260 & -0.23490 & 0.00226 & 0.20311 & 0.07977 & -0.00042 & -0.19364 & -0.00104 \\
\hline $\mathrm{x} 19$ & 0.05062 & 0.00016 & -0.04717 & -0.00014 & 0.00028 & 0.01482 & 0.00059 & 0.00994 \\
\hline $\mathrm{x} 20$ & -0.05676 & -0.00017 & 0.05290 & 0.00016 & -0.00032 & -0.01662 & -0.00066 & -0.01115 \\
\hline $\mathrm{x} 21$ & 0.19681 & -0.00253 & -0.18170 & 0.00225 & 0.00110 & 0.08317 & -0.00038 & 0.00511 \\
\hline x22 & -0.21899 & 0.00281 & 0.20217 & -0.00250 & -0.00123 & -0.09255 & 0.00042 & -0.00568 \\
\hline$x 23$ & 0.00237 & -0.09773 & -0.00232 & 0.09049 & 0.01981 & 0.00094 & -0.07489 & -0.00031 \\
\hline $\mathrm{x} 24$ & -0.00263 & 0.10862 & 0.00258 & -0.10058 & -0.02202 & -0.00105 & 0.08324 & 0.00035 \\
\hline$x 25$ & -0.00015 & -0.00139 & -0.00011 & 0.00145 & -0.00512 & $6.83515 \mathrm{e}-05$ & -0.00769 & 0.00074 \\
\hline
\end{tabular}


$\mathrm{H}=$

\begin{tabular}{|c|c|c|c|c|c|c|c|c|c|c|}
\hline & $\mathrm{x} 1$ & $\mathrm{x} 2$ & $\mathrm{x} 3$ & $\mathrm{x} 4$ & $\mathrm{x} 5$ & $\mathrm{x} 6$ & $\mathrm{x} 7$ & $\mathrm{x} 8$ & $\mathrm{x} 9$ & $\mathrm{x} 10$ \\
\hline y1 & 0.06039 & 0.05370 & -0.22883 & -0.22715 & 0.67060 & 0.59120 & -0.00026 & -0.00025 & 0.00035 & 0.00036 \\
\hline y2 & 0.00081 & 0.00075 & 0.00016 & 0.00016 & -0.00010 & $-9.37500 e-05$ & -5.71803 & -5.65955 & 2.00886 & 1.99237 \\
\hline y3 & 0.00520 & 0.00368 & -0.18160 & -0.18004 & -0.00571 & -0.00411 & 0.00031 & $0.00032-8$. & $93054 \mathrm{e}-05-8$ & $8.15625 \mathrm{e}-05$ \\
\hline y4 & -0.00029 & -0.00027 & 0.00011 & 0.00011 & $2.33371 \mathrm{e}-05$ & $2.11047 \mathrm{e}-05$ & -1.04028 & -1.02964 & 3.27399 & 3.24713 \\
\hline y5 & -0.47634 & -0.44177 & -0.01295 & -0.01292 & 0.04555 & 0.04120 & -0.64212 & -0.64133 & -0.16474 & -0.16456 \\
\hline y6 & -0.04246 & -0.03911 & -0.11239 & -0.11099 & -0.58510 & -0.50858 & 0.00185 & 0.00185 & 0.00176 & 0.00175 \\
\hline y7 & -0.47169 & -0.43685 & -0.01281 & -0.01279 & 0.04518 & 0.04080 & -1.47188 & -1.46229 & -0.41584 & -0.41361 \\
\hline y8 & 0.02862 & 0.02440 & -0.13392 & -0.13243 & 0.30918 & 0.26660 & 0.00264 & 0.00263 & 0.00323 & 0.00322 \\
\hline y9 & 0.52405 & 0.56535 & 0.01515 & 0.01518 & -0.04387 & -0.04851 & 0.55225 & 0.55795 & 0.14007 & 0.14123 \\
\hline y 10 & -0.05893 & -0.06358 & 0.06401 & 0.06413 & -0.60703 & -0.67124 & 0.00224 & 0.00226 & 0.00147 & 0.00149 \\
\hline
\end{tabular}

(H continued)

$\begin{array}{lcccccccccc} & \mathrm{x} 11 & \mathrm{x} 12 & \mathrm{x} 13 & \mathrm{x} 14 & \mathrm{x} 15 & \mathrm{x} 16 & \mathrm{x} 17 & \mathrm{x} 18 & \mathrm{x} 19 & \mathrm{x} 20 \\ \mathrm{y} 1 & 0.56271 & 0.57102 & 4.43621 & 4.40772 & -5.16187 & -5.15139 & -0.18582 & -0.18344 & -4.27285 & -4.26445 \\ \mathrm{y} 2 & 0.01776 & 0.01766 & -0.03425 & -0.03407 & -0.14524 & -0.14495 & -16.86753 & -16.65300 & -0.01301 & -0.01298 \\ \mathrm{y} 3 & -0.34129 & -0.32732 & -1.57622 & -1.56933 & 4.00242 & 3.99411 & 0.16251 & 0.16044 & 3.99399 & 3.98619 \\ \mathrm{y} 4 & 0.01263 & 0.01256 & -0.00510 & -0.00507 & 0.12531 & 0.12506 & 14.58607 & 14.40055 & 0.01162 & 0.01160 \\ \mathrm{y} 5 & 0.00493 & 0.00492 & 0.01349 & 0.01343 & 0.08254 & 0.08239 & 6.00499 & 5.93567 & -0.02490 & -0.02485 \\ \text { y6 } & 2.68857 & 2.68542 & 3.37164 & 3.36095 & -4.76086 & -4.75207 & -0.02577 & -0.02528 & -1.15055 & -1.14789 \\ \text { y7 } & 0.00750 & 0.00747 & -0.03003 & -0.02989 & -0.14120 & -0.14092 & -14.13047 & -13.95656 & -0.05022 & -0.05012 \\ \text { y8 } & 8.48140 & 8.44335 & -11.83824 & -11.78361 & 2.36429 & 2.35972 & -0.07771 & -0.07680 & -0.86131 & -0.85970 \\ \text { y9 } & -0.00139 & -0.00140 & -0.00661 & -0.00664 & -0.02261 & -0.02266 & -1.69895 & -1.72084 & 0.00667 & 0.00669 \\ \text { y10 } & 1.89677 & 1.90807 & 0.94343 & 0.94827 & 0.07363 & 0.07377 & 0.02501 & 0.02533 & 0.44253 & 0.44342\end{array}$


(H continued)

$\begin{array}{lcccccc} & \mathrm{x} 21 & \mathrm{x} 22 & \mathrm{x} 23 & \mathrm{x} 24 & \mathrm{x} 25 & \mathrm{x} 26 \\ \text { y1 } & -16.93617 & -16.73085 & -0.23240 & -0.22604 & 0.01666 & 0.01662 \\ \text { y2 } & 0.21653 & 0.21388 & 9.49150 & 9.22643 & 0.16160 & 0.16128 \\ \text { y3 } & 15.62219 & 15.43242 & 0.22744 & 0.22119 & 0.01254 & 0.01251 \\ \text { y4 } & -0.19277 & -0.19041 & -8.78962 & -8.54415 & -0.16838 & -0.16804 \\ \text { y5 } & -0.09916 & -0.09805 & -1.93200 & -1.87890 & 0.59600 & 0.59482 \\ \text { y6 } & -6.97286 & -6.88380 & -0.09679 & -0.09396 & -0.00803 & -0.00801 \\ \text { y7 } & 0.03262 & 0.03222 & 7.36773 & 7.16734 & 0.89653 & 0.89475 \\ \text { y8 } & -0.50380 & -0.49926 & 0.03011 & 0.02927 & -0.08707 & -0.08690 \\ \text { y9 } & 0.01554 & 0.01573 & 0.26361 & 0.27119 & 0.00515 & 0.00516 \\ \text { y10 } & 1.12595 & 1.13991 & 0.00844 & 0.00869 & -0.00034 & -0.00034\end{array}$

$\mathrm{J}=$

$\begin{array}{lcccccccc} & \mathrm{u} 1 & \mathrm{u} 2 & \mathrm{u} 3 & \mathrm{u} 4 & \mathrm{u} 5 & \mathrm{u} 6 & \mathrm{u} 7 & \mathrm{u} 8 \\ \mathrm{y} 1 & 1.35144 & -0.00177 & -0.24938 & 0.00094 & 0.00045 & 0.09631 & 0.00263 & 0.14059 \\ \mathrm{y} 2 & -0.00186 & 1.32377 & 0.00140 & -0.28714 & -0.15331 & -0.00129 & 0.61368 & 0.00507 \\ \text { y3 } & -0.24933 & 0.00144 & 0.68350 & -0.00187 & -0.00075 & -0.06460 & -0.00251 & 0.14878 \\ \text { y4 } & 0.00088 & -0.28718 & -0.00187 & 0.68424 & 0.16752 & 0.00126 & -0.42558 & 8.00020 \mathrm{e}-05 \\ \text { y5 } & 0.00043 & -0.15498 & -0.00076 & 0.16838 & 0.27673 & 0.00065 & 0.06726 & -0.00086 \\ \text { y6 } & 0.09703 & -0.00135 & -0.06570 & 0.00134 & 0.00052 & 0.72447 & 0.00085 & -0.03067 \\ \text { y7 } & 0.00256 & 0.61461 & -0.00263 & -0.42630 & 0.06817 & 0.00090 & 0.63961 & 0.00327 \\ \text { y8 } & 0.13758 & 0.00515 & 0.15072 & 5.07019 \mathrm{e}-05 & -0.00082 & -0.03063 & 0.00318 & 1.63649 \\ \text { y9 } & -8.75739 \mathrm{e}-05 & 0.00868 & -4.30644 \mathrm{e}-05 & -0.00575 & 0.00754 & 2.91297 \mathrm{e}-05 & -0.01235 & -0.00018 \\ \text { y10 } & 0.00044 & -0.00015 & -0.00124 & 0.00024 & -0.00015 & -0.00262 & 0.00039 & 0.00023\end{array}$

» diary 


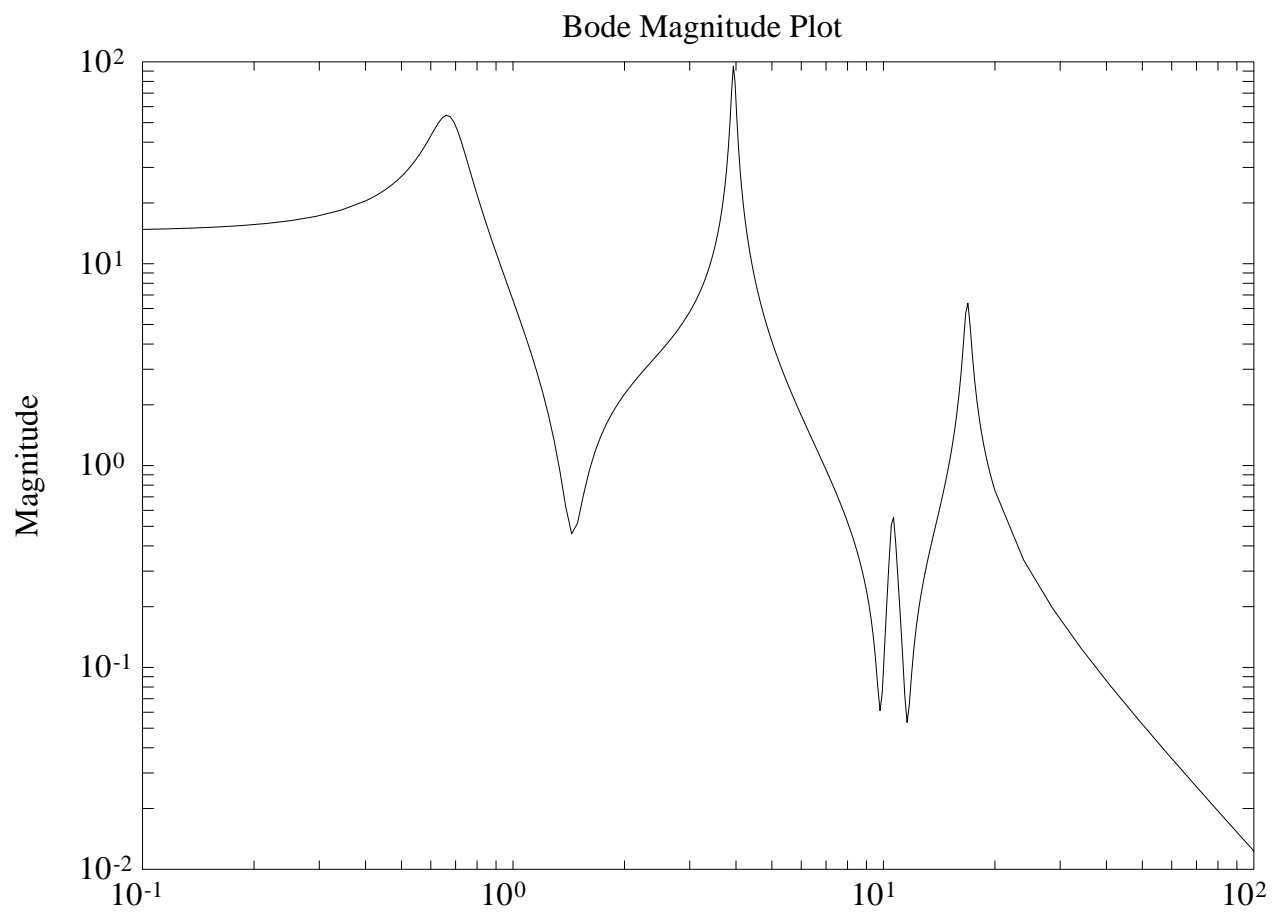

Freq. rad/s

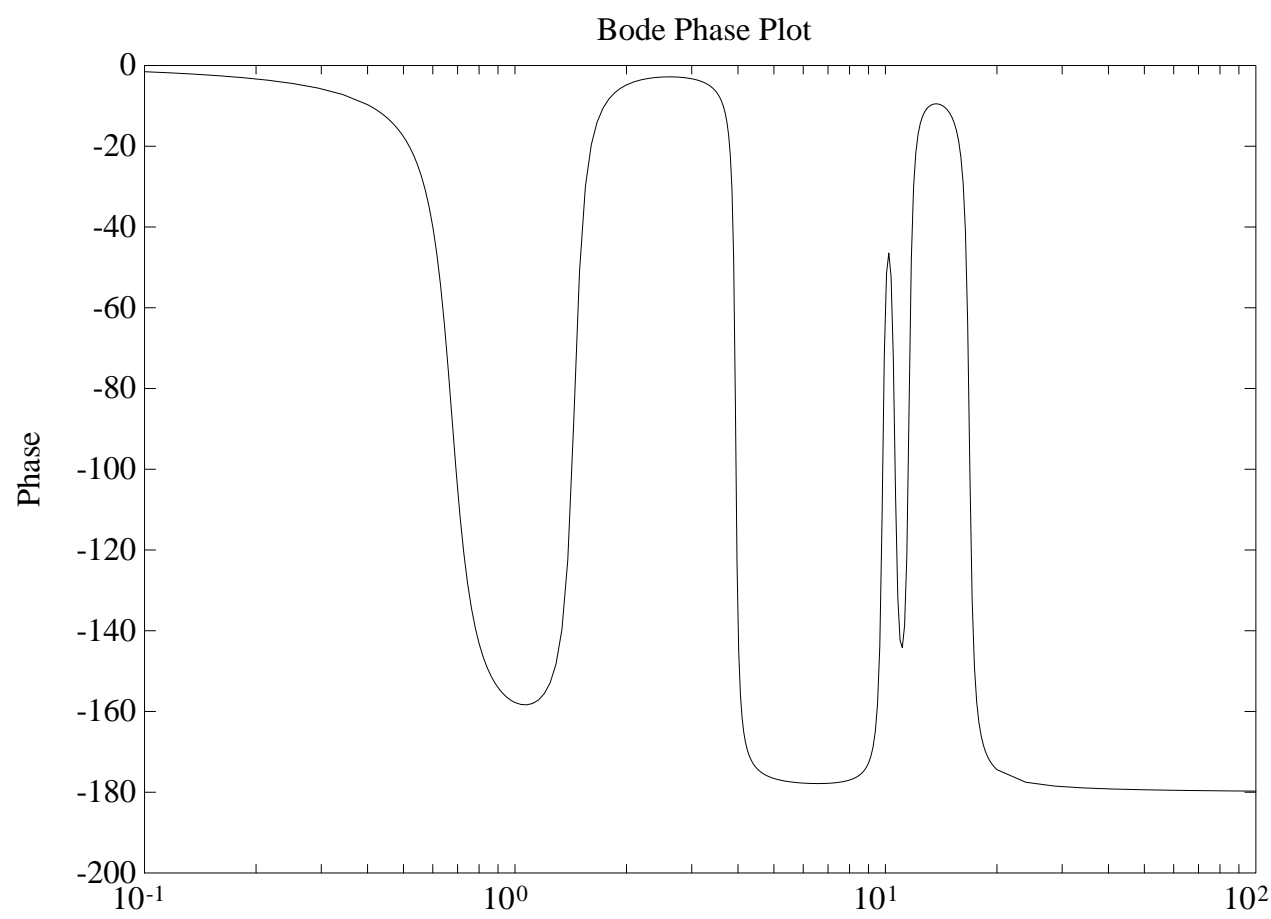

Freq. rad/s

Figure 1.1 Bode Magnitude and Phase Plots of Original Order 8 System. 


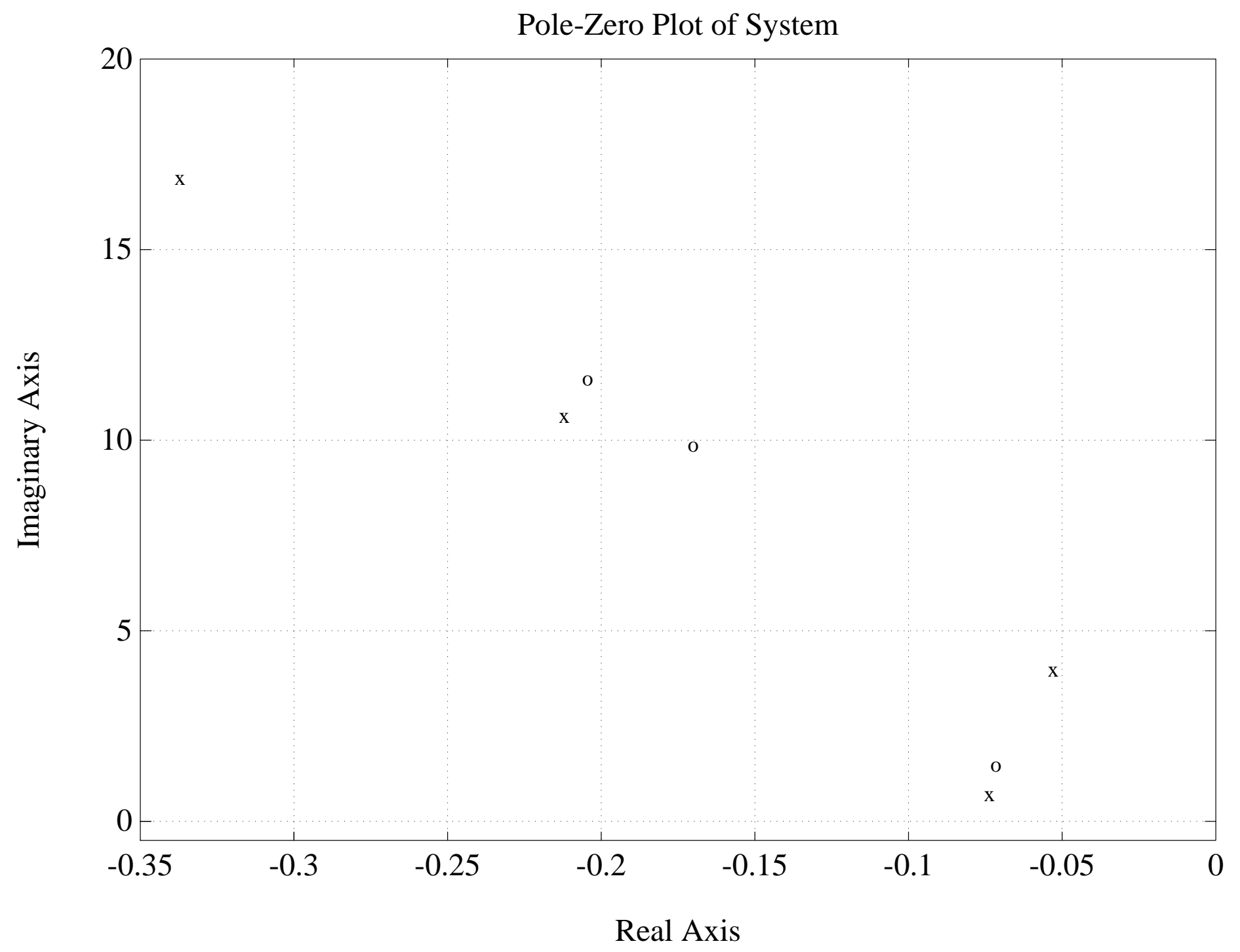

Figure 1.2 Page 44. Pole-Zero Plot of Original Order 8 System. Poles marked by X, and zeros marked by O. 


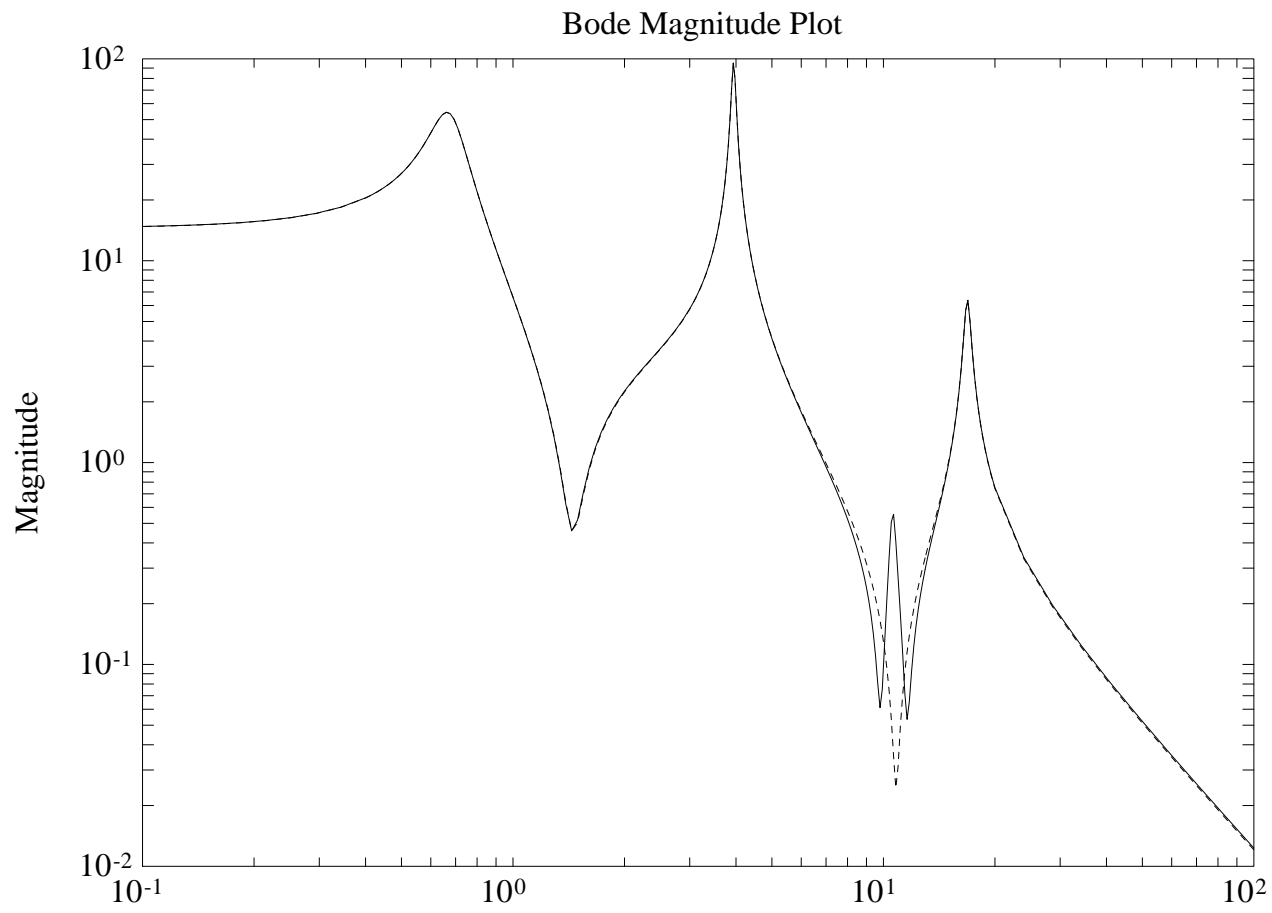

Freq. rad/s

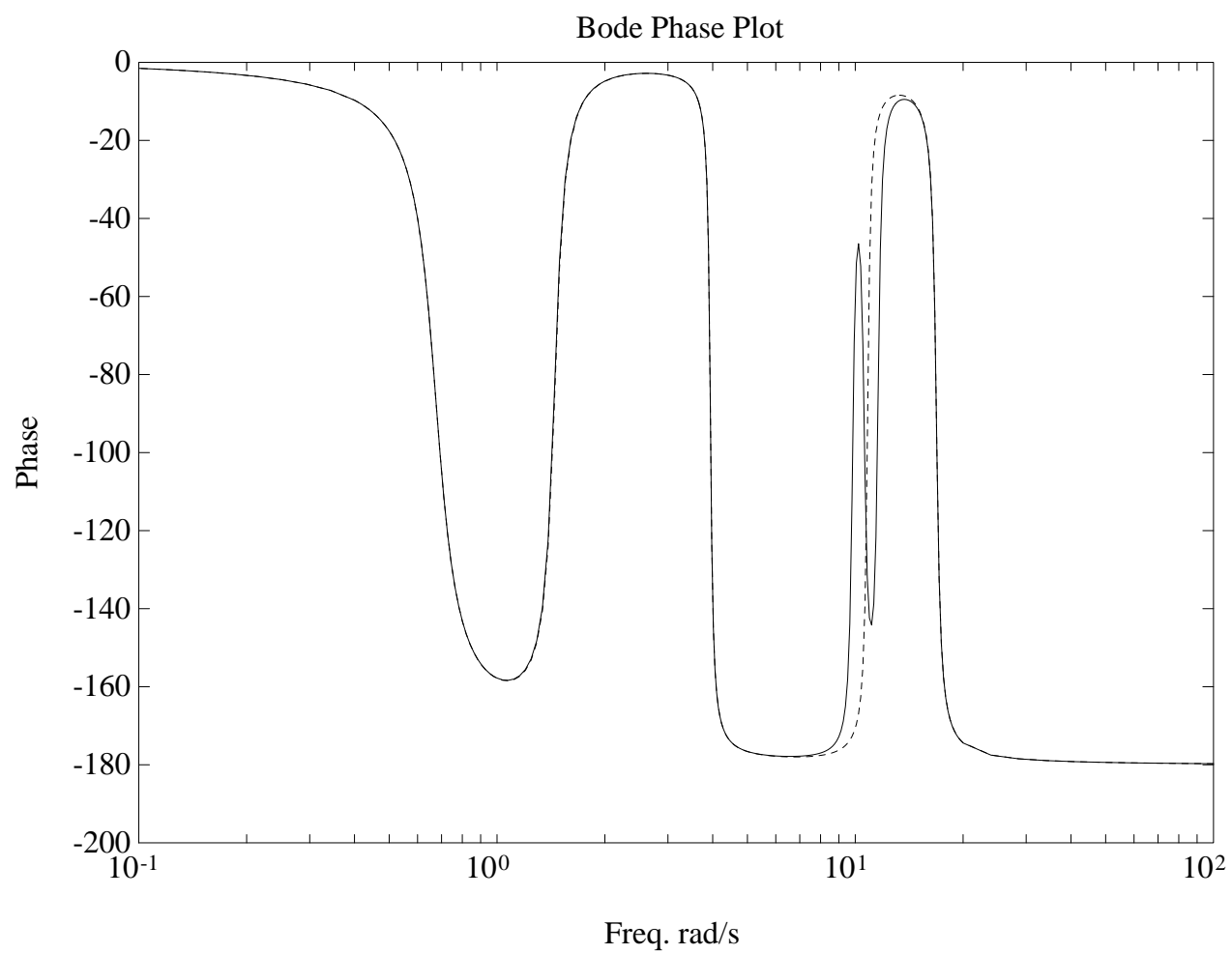

Figure 1.3 Bode Plots of Reduced Order 6 System. Matrix Pencil Approach in dashed line; full order model in solid line. 


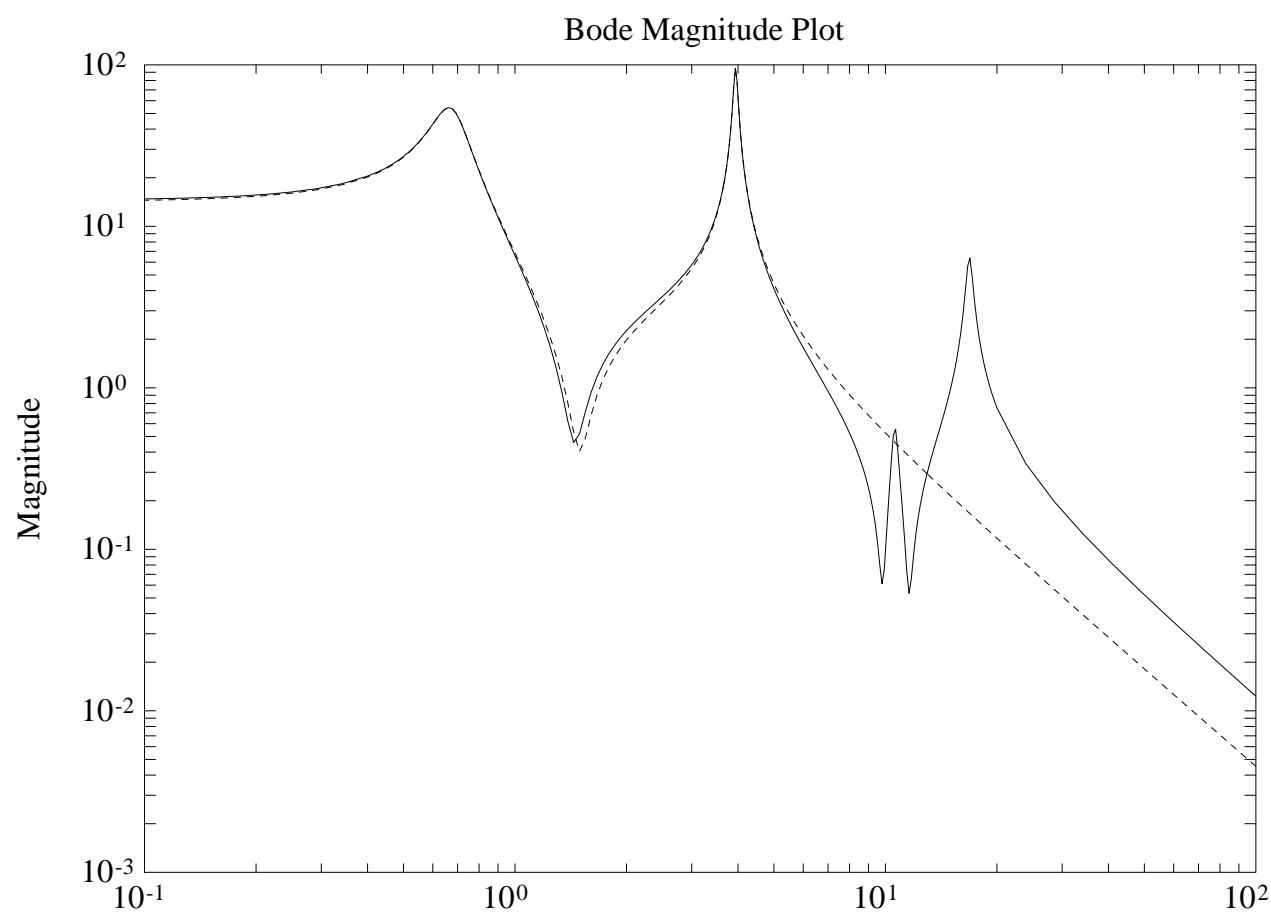

Freq. rad/s

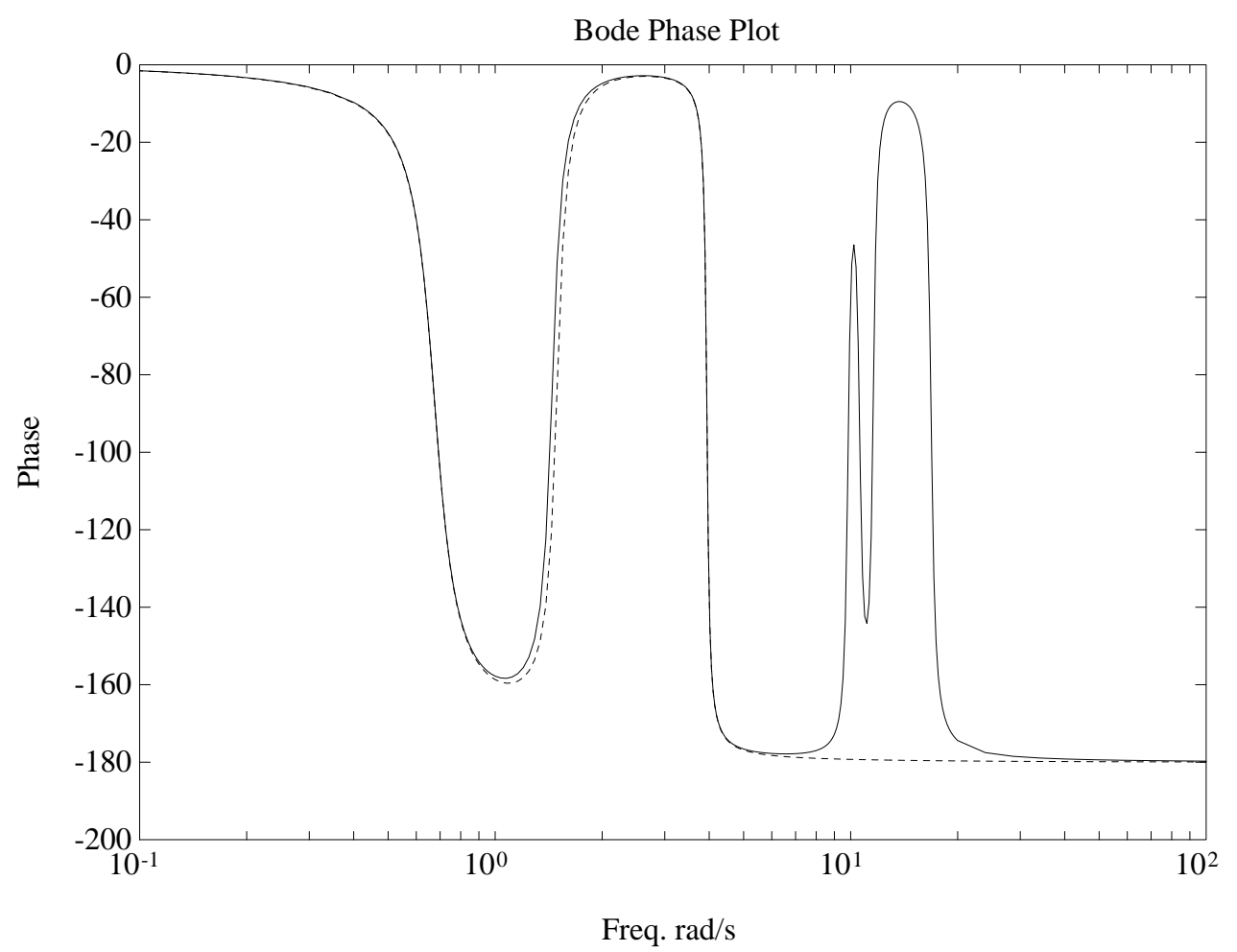

Figure 1.4 Bode Plots of Reduced Order 4 System. Matrix Pencil Approach in dashed line; full order model in solid line. 


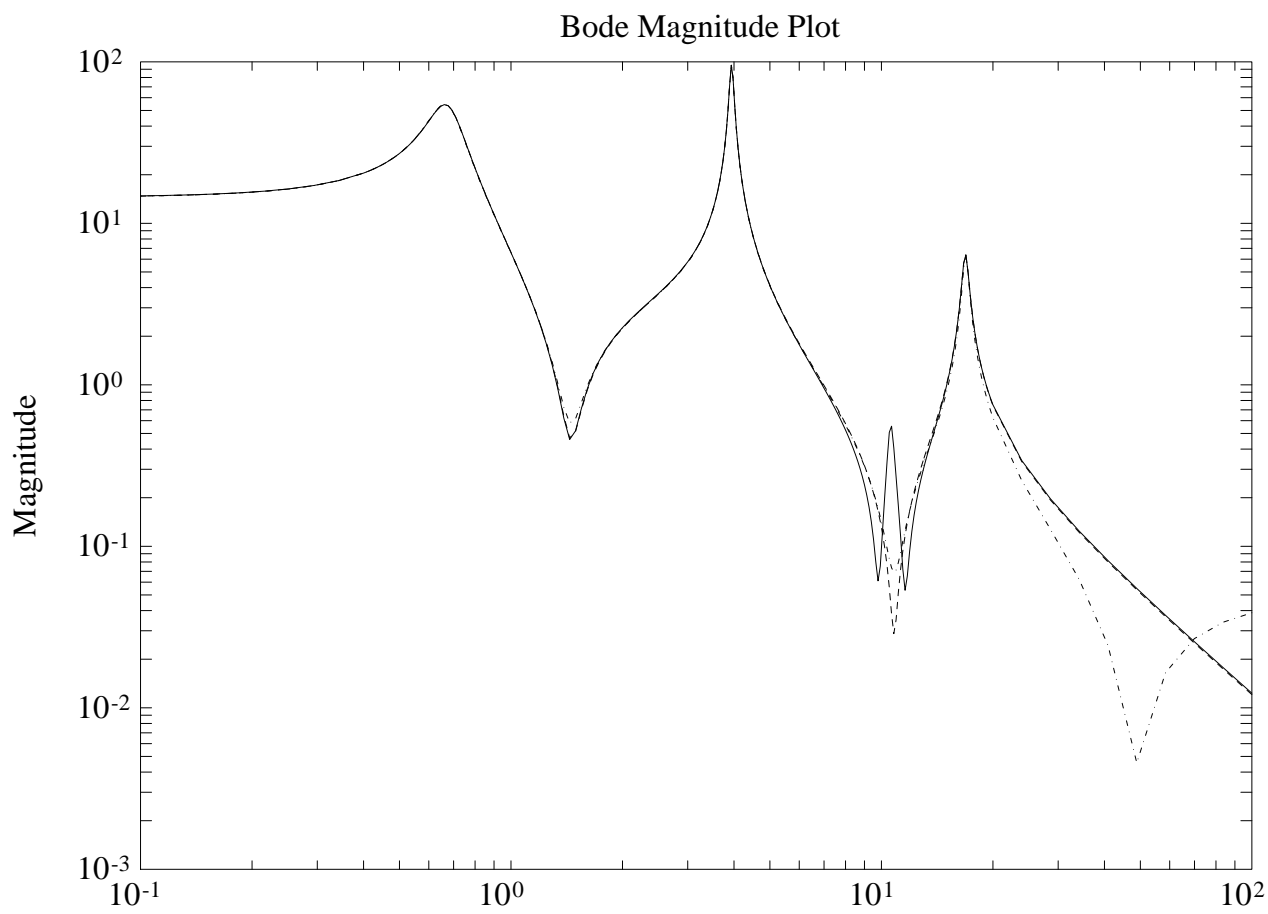

Freq. rad/s

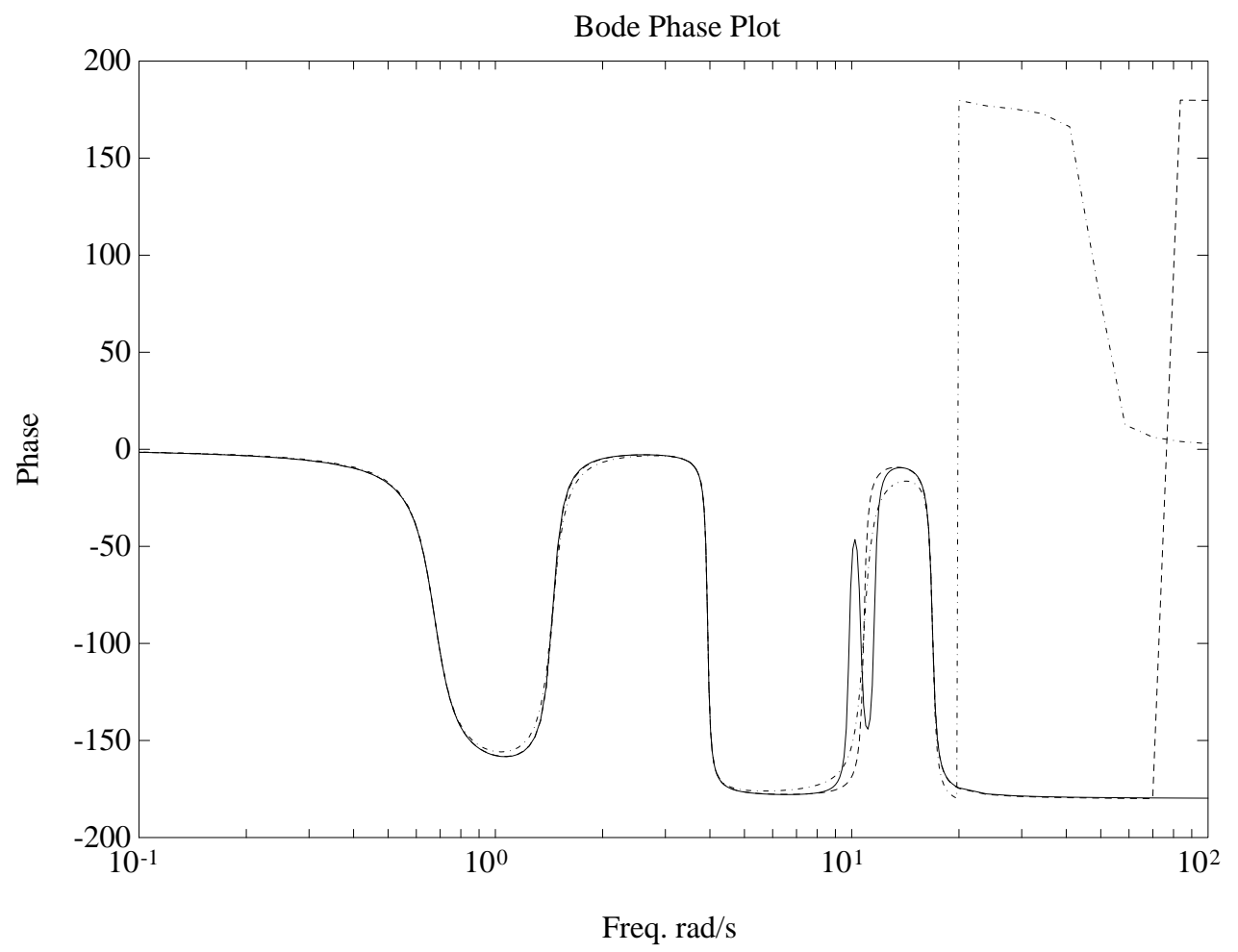

Figure 1.5 Bode Plots of Reduced Order 6; Balanced Realization (dashed), and Hankel Norm (dashdot) Methods. Full order model in solid line. 


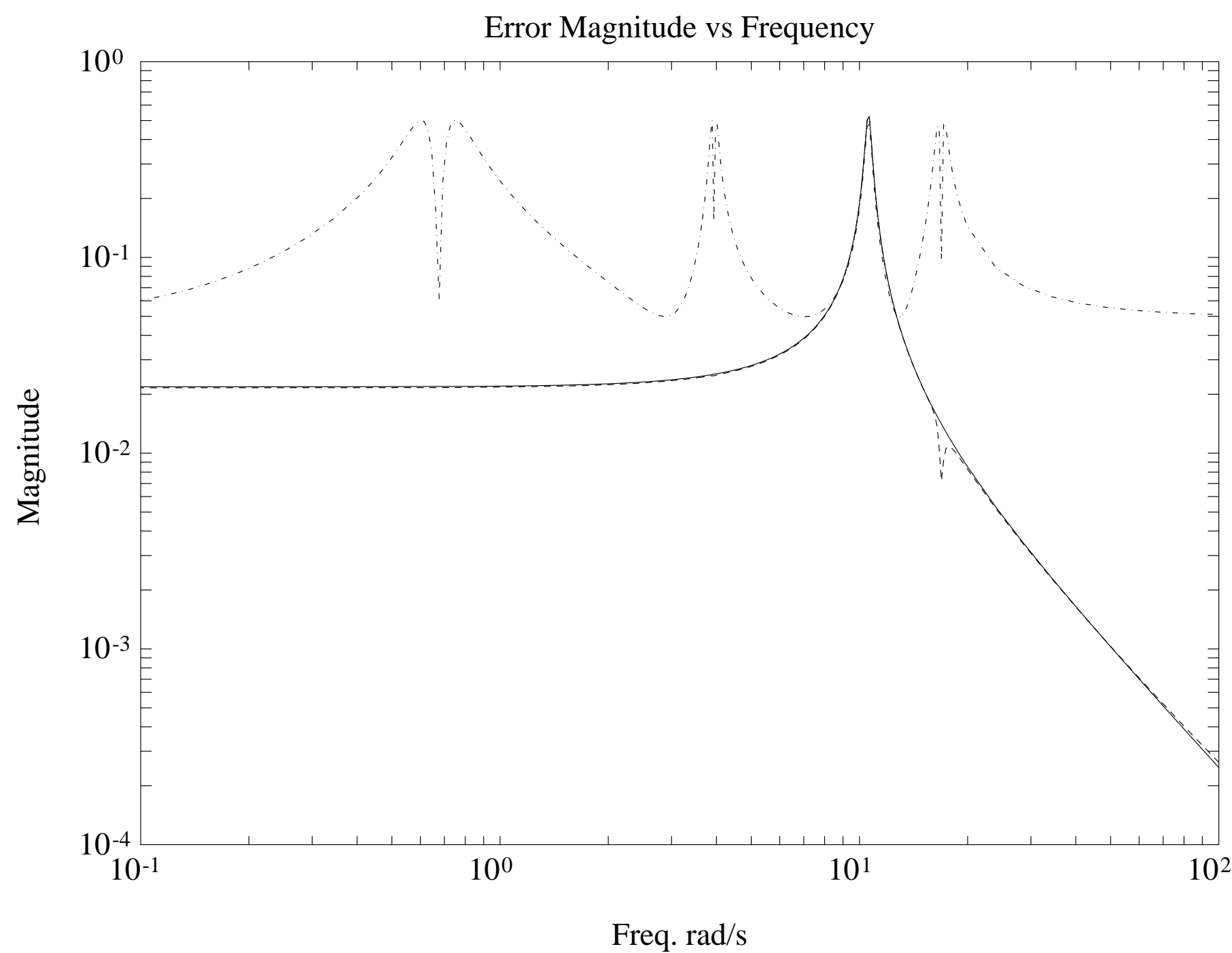

Figure 1.6 Page 48. Error Magnitude Plots for Reduced Order 6 Systems. Matrix Pencil in solid line, Balanced Realization in dashed line, and Hankel Norm in dashdot line. 


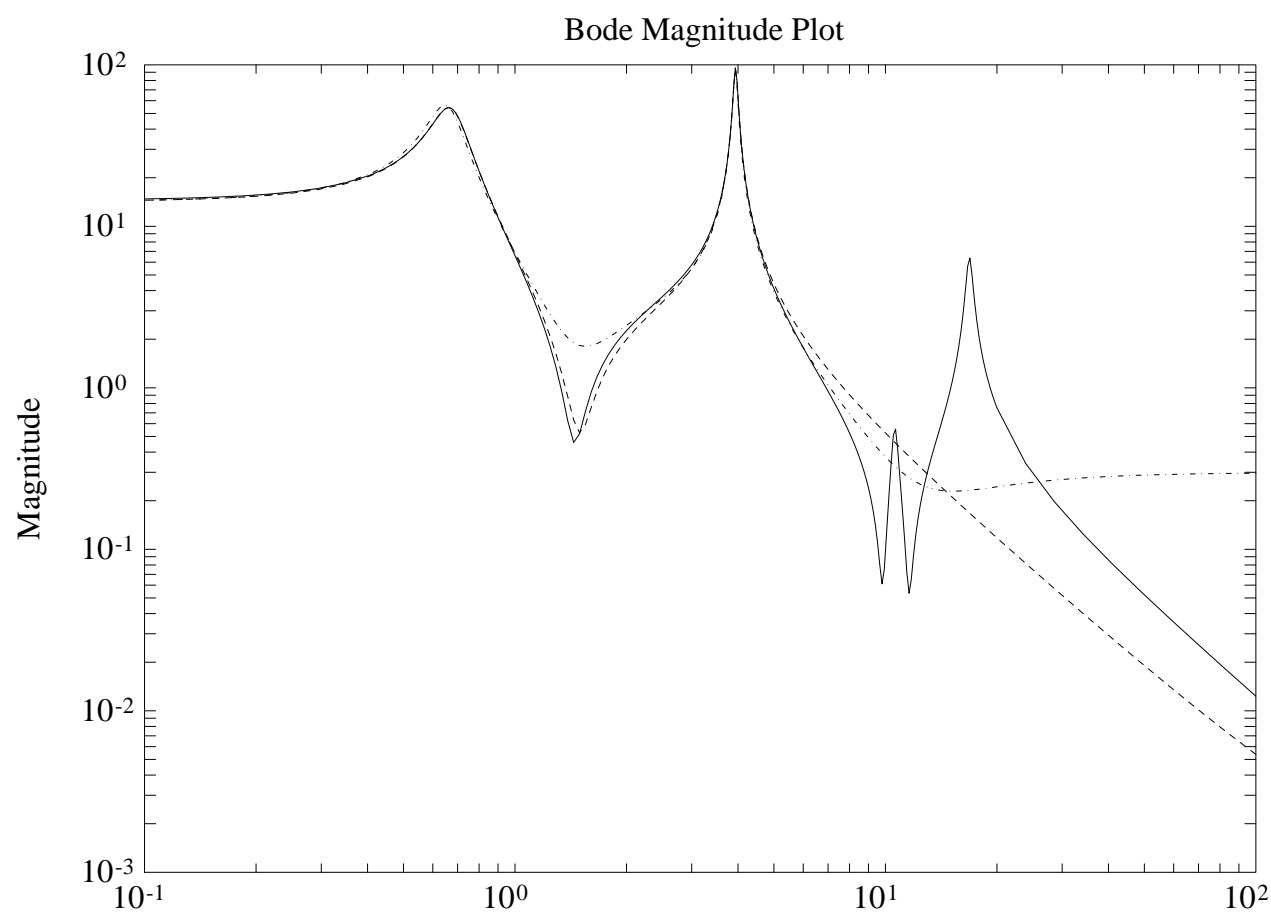

Freq. rad/s

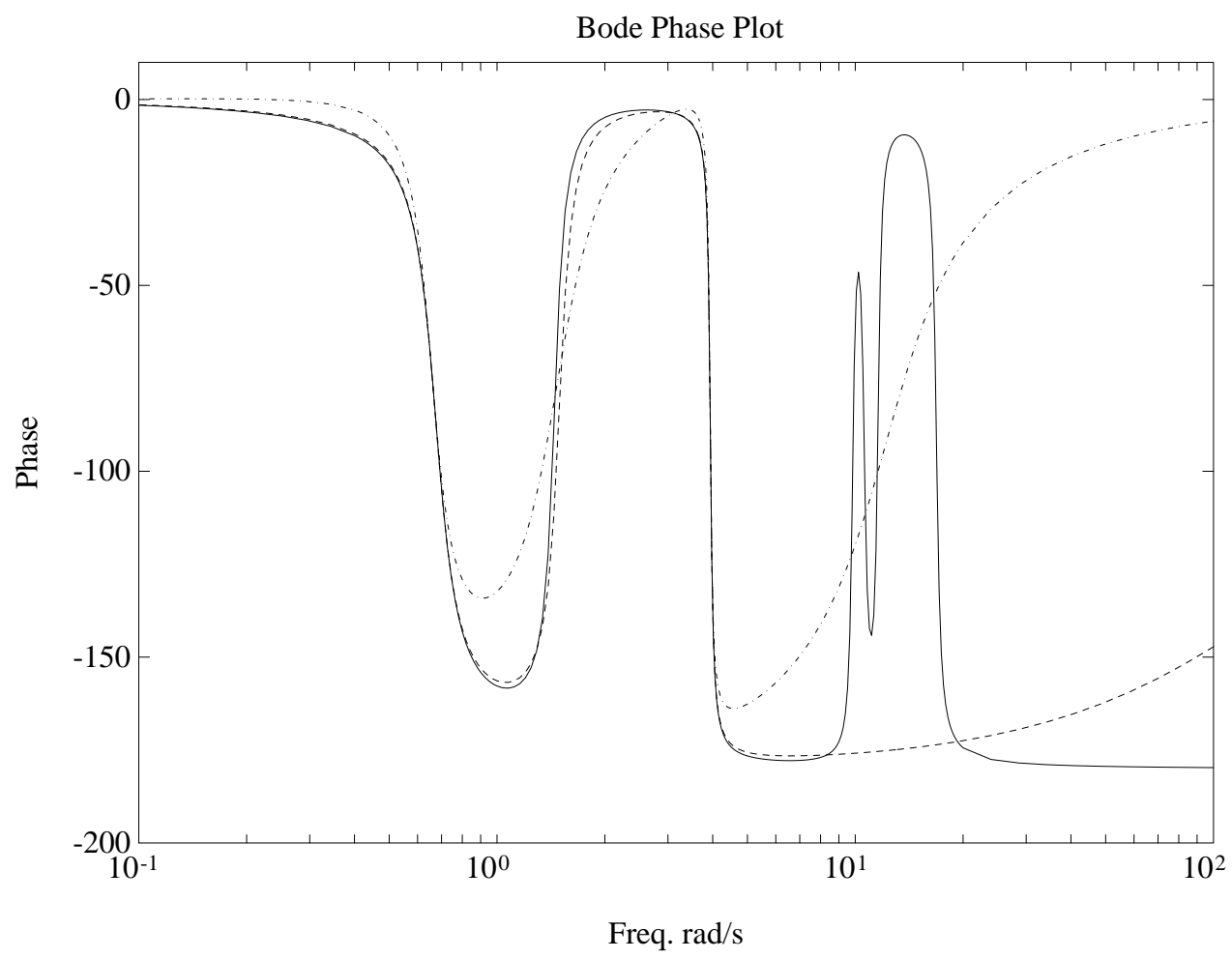

Figure 1.7 Bode Plots of Reduced Order 4 Systems; Balanced Realization (dashed) and Hankel Norm (dashdot) Methods. Full order model in solid line. 


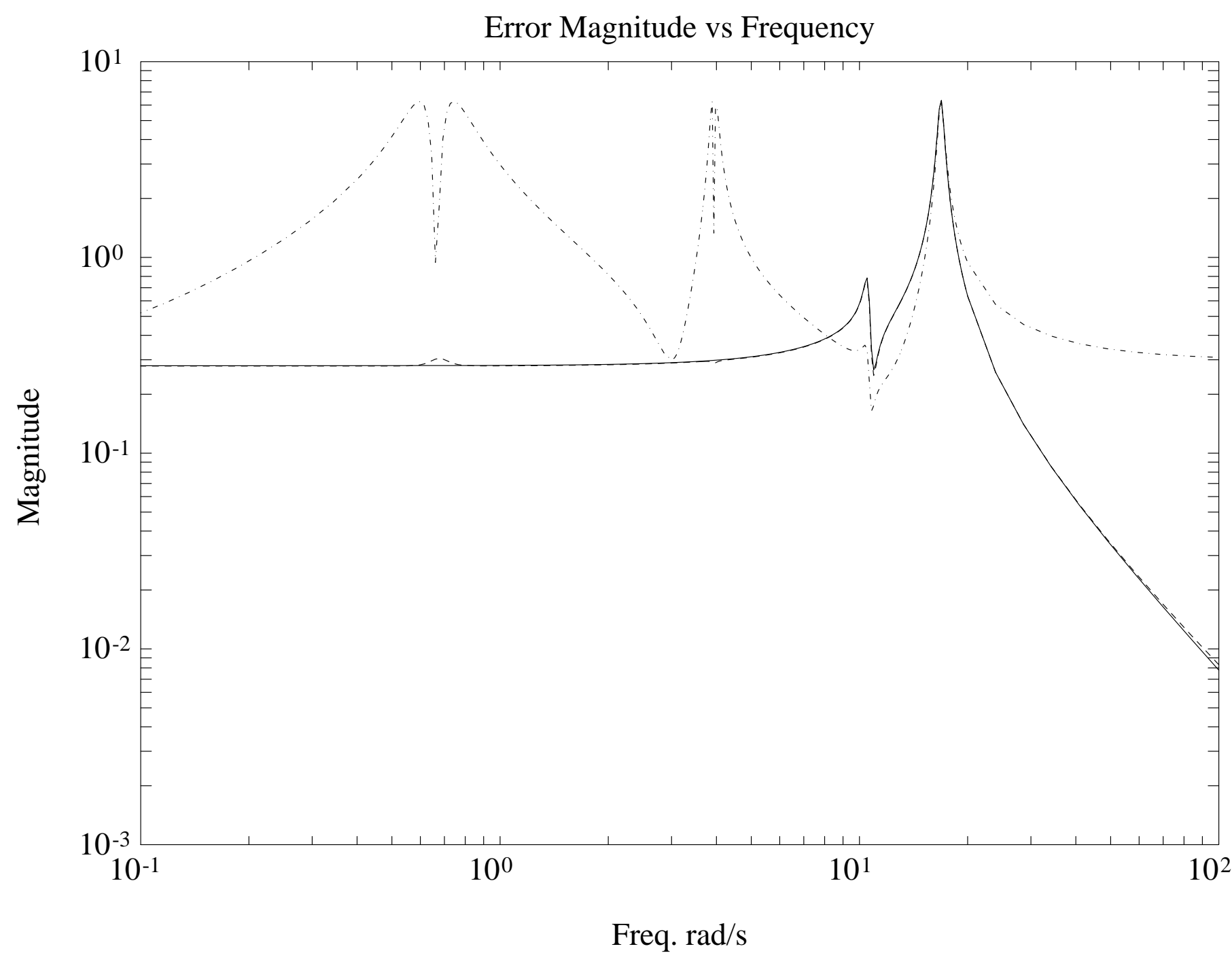

Figure 1.8 Page 50. Error Magnitude Plots for Reduced Order 4 Systems. Matrix Pencil approach in solid line, Balanced Realization in dashed line, and Optimal Hankel norm in dashdot line. 


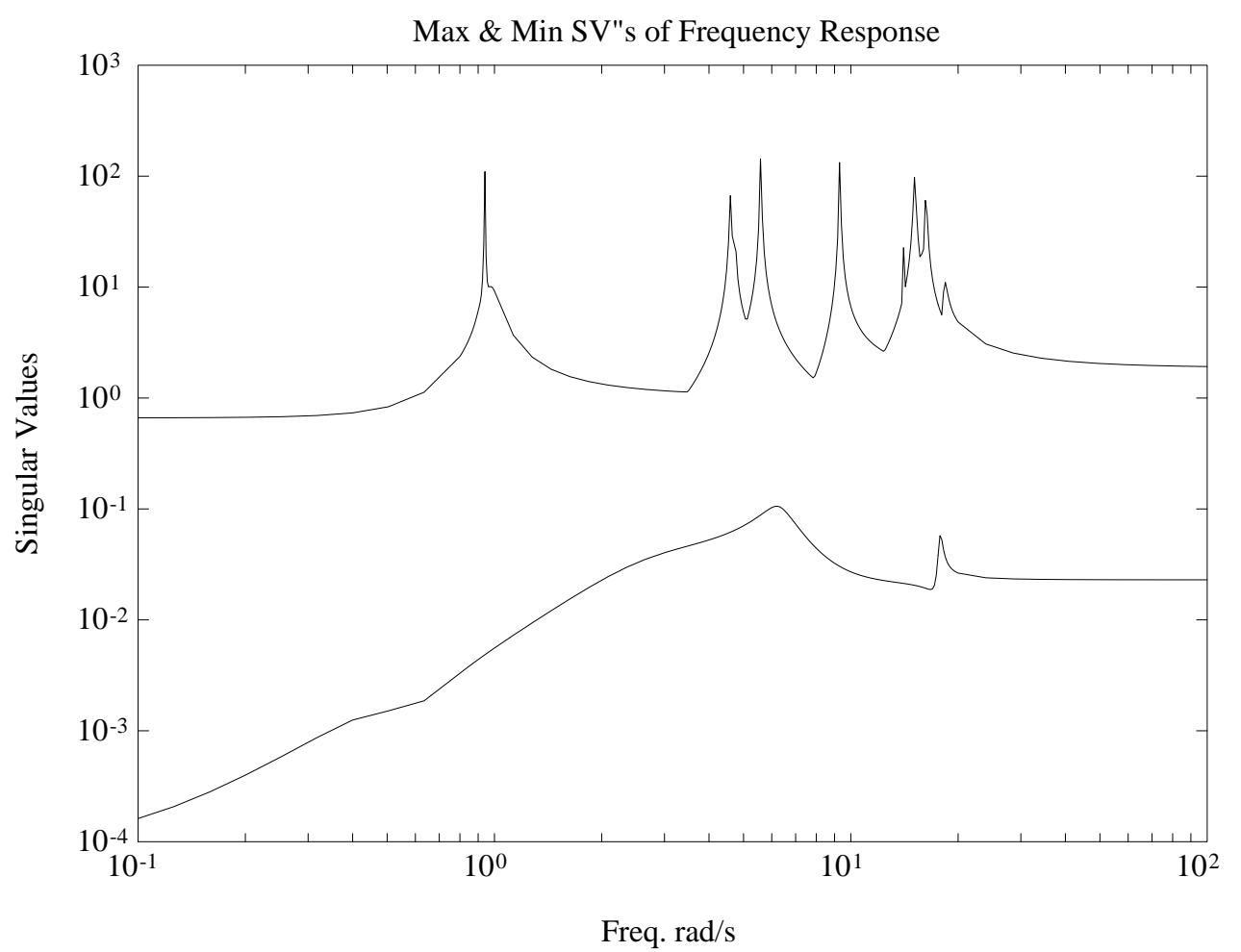

Figure 2.1 Max \& Min SV's of Original Order 26 System.

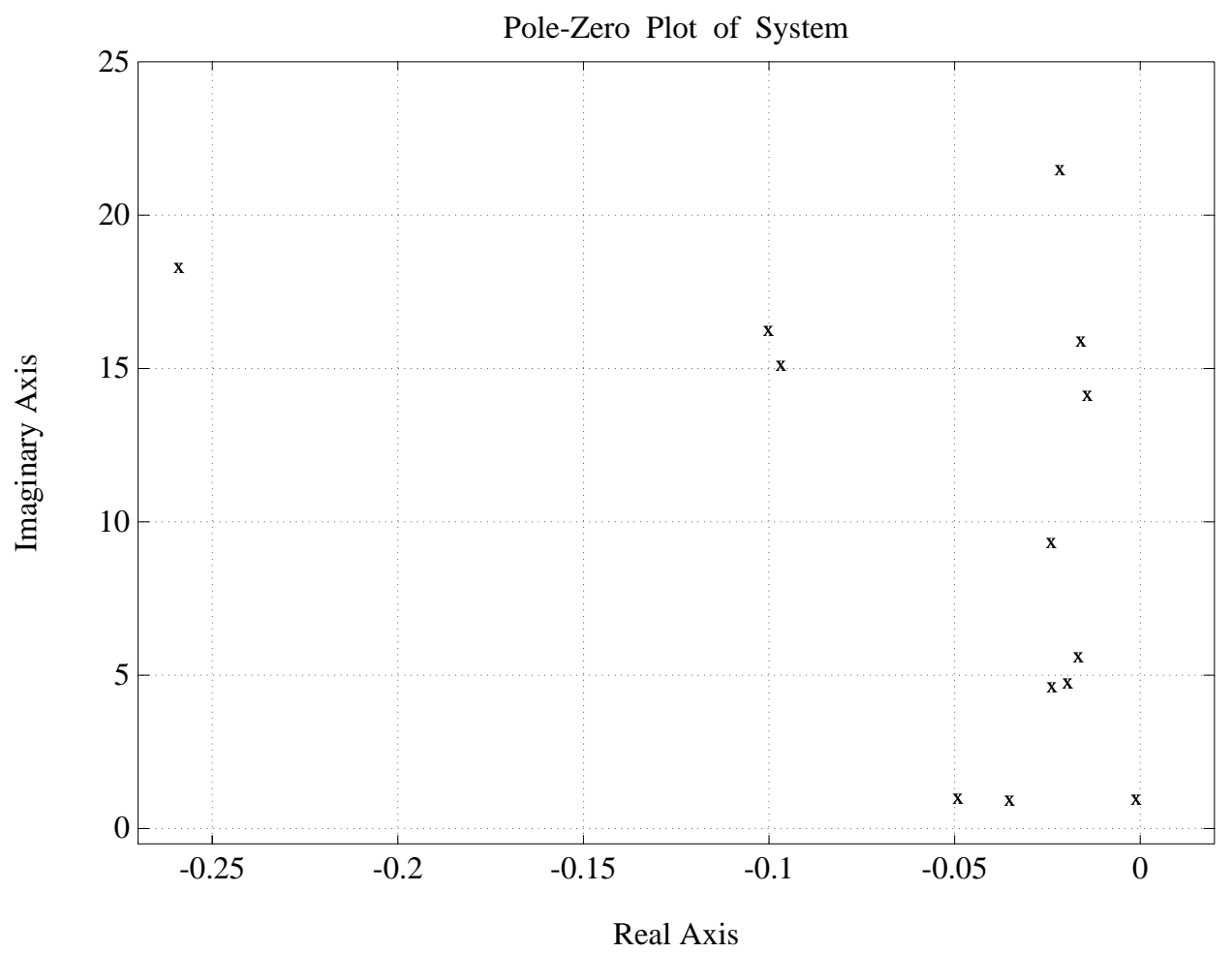

Figure 2.2 Pole-Zero plot of Original Order 26 System. Poles marked by X, and zeros by O. 


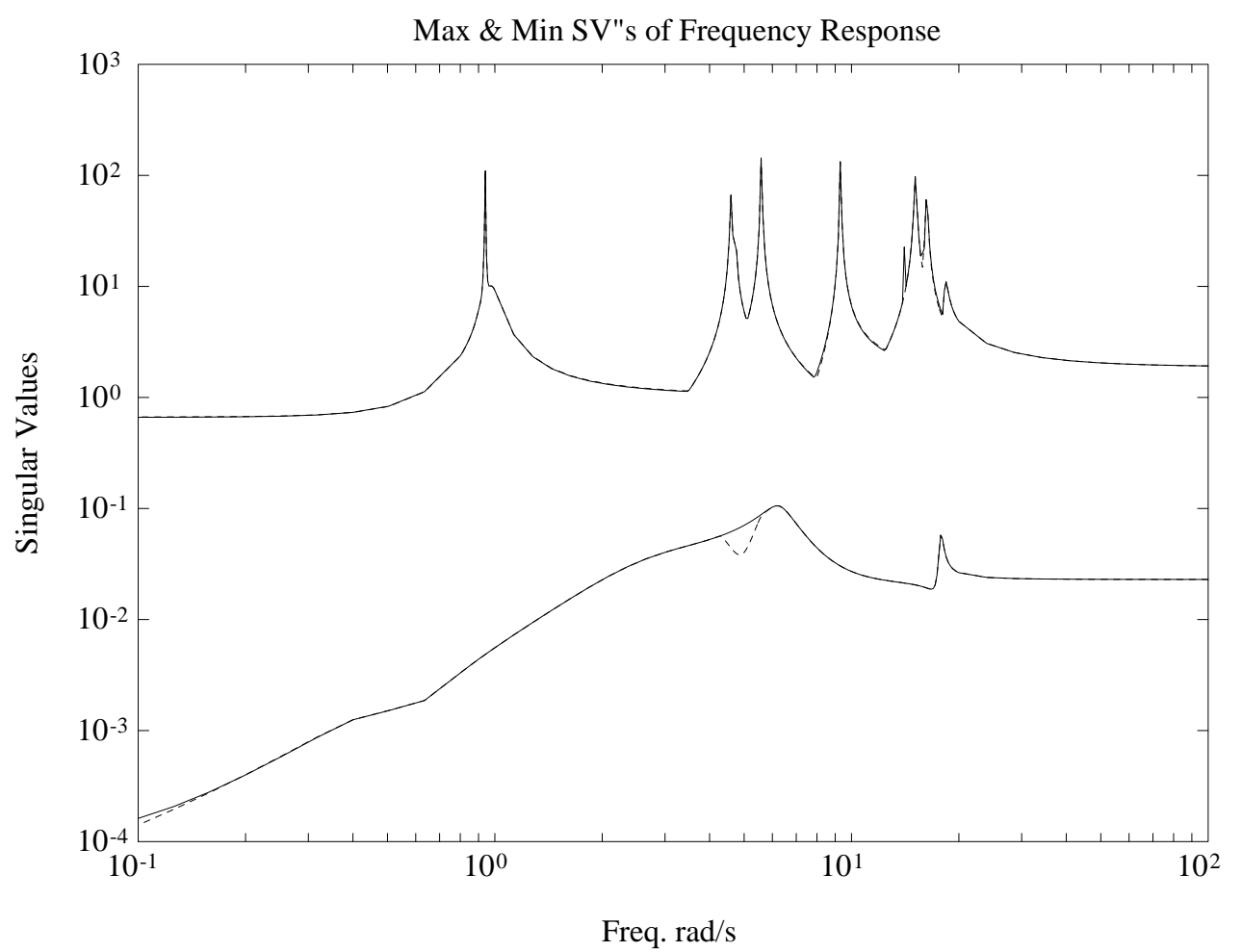

Figure 2.3 Max \& Min SV's of Reduced Order 20 System; Matrix Pencil Approach in dashed line. Full order model in solid line.

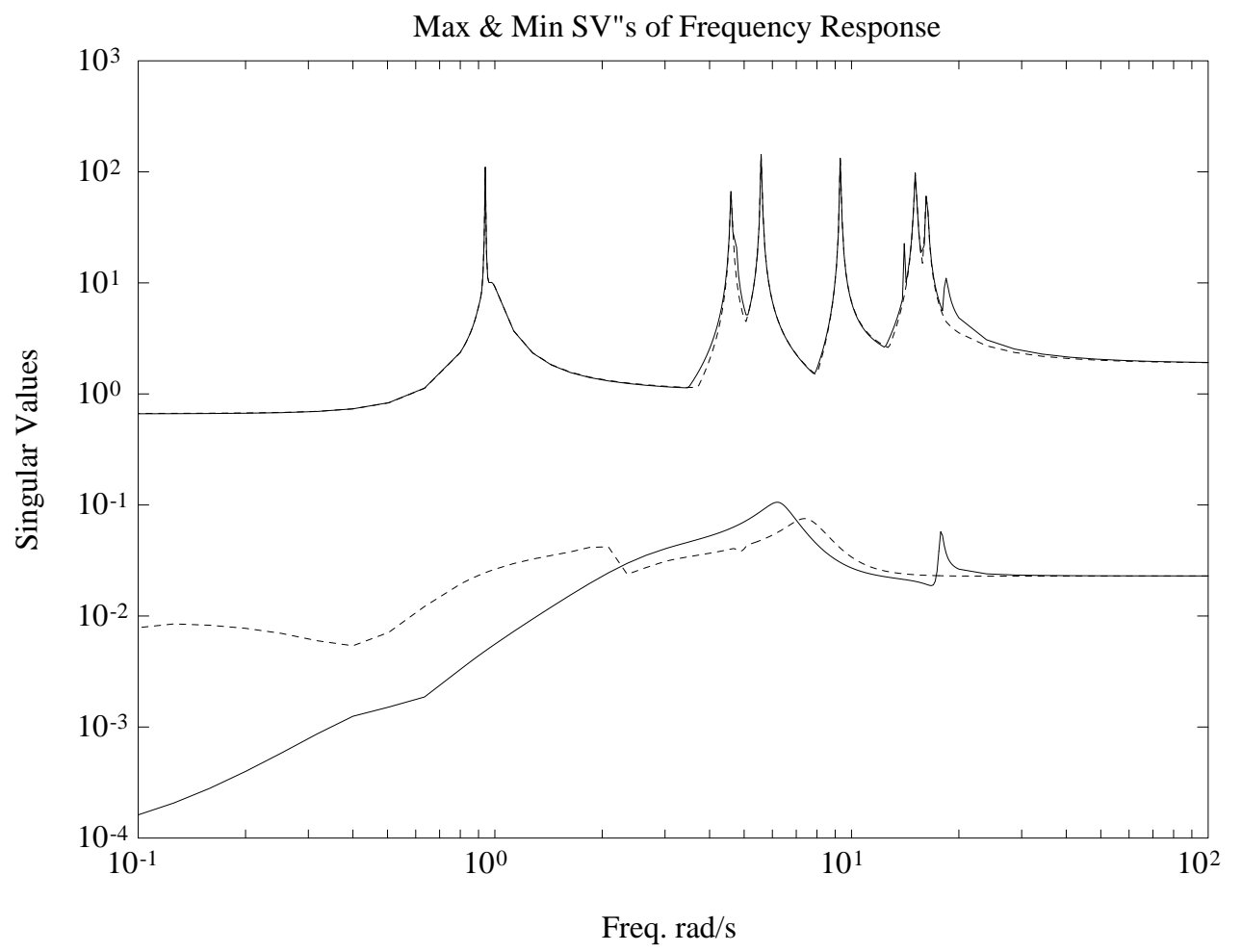

Figure 2.4 Max \& Min SV's of Reduced Order 16 Model; Matrix Pencil Approach in dashed line. Full order model in solid line. 


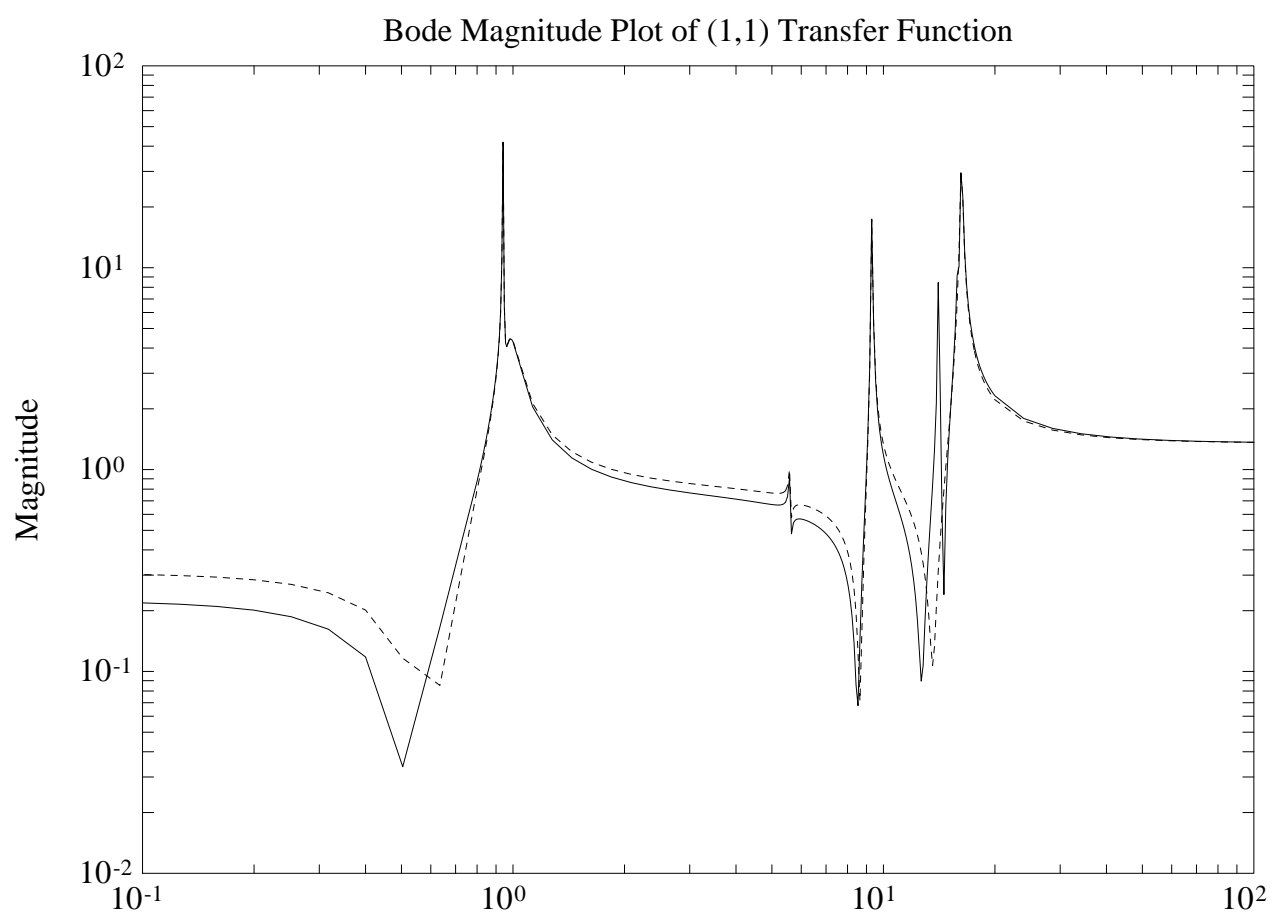

Freq. rad/s

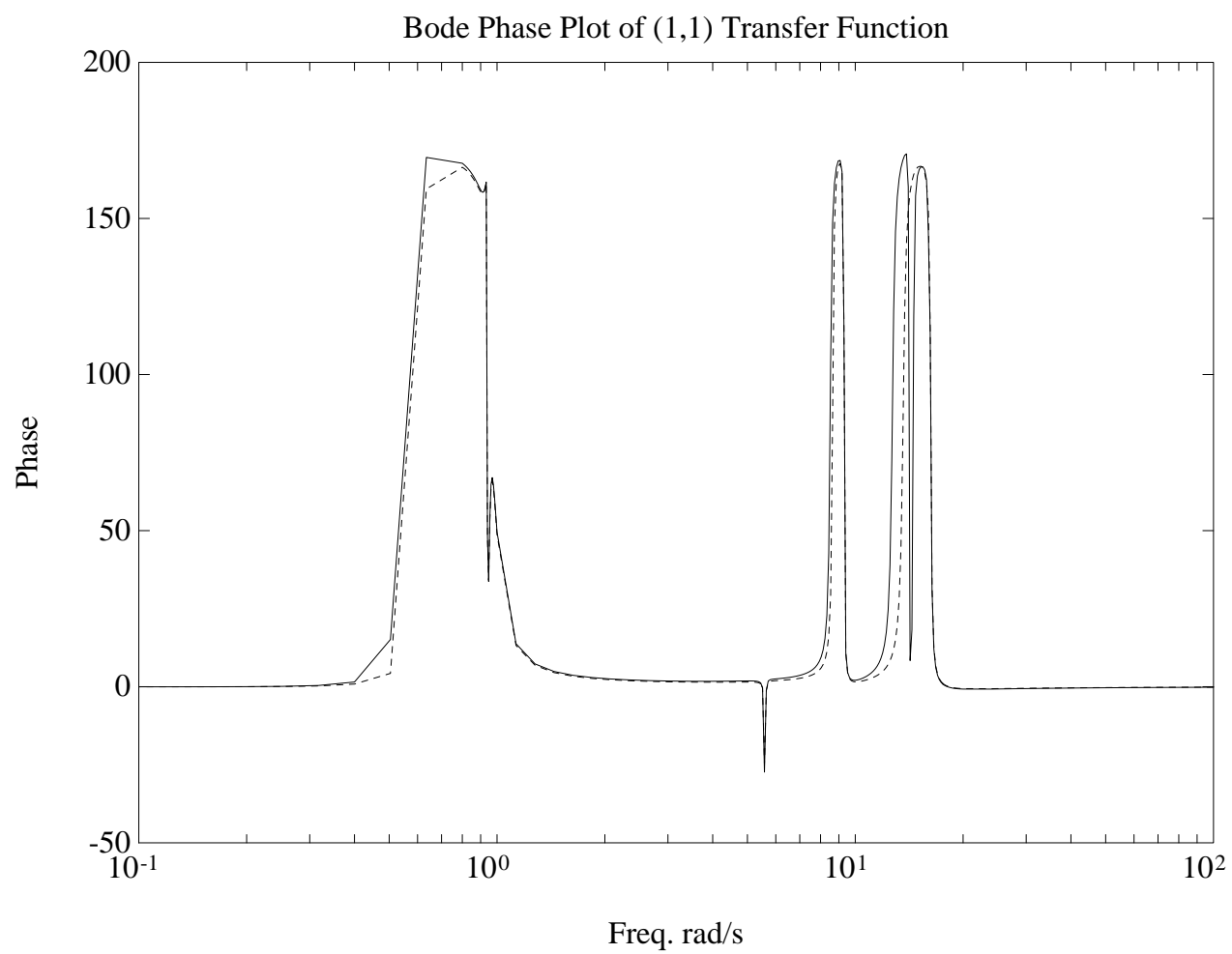

Figure 2.5 Bode Plots of Reduced Order 20 of $(1,1)$ Transfer Function; Matrix Pencil Approach in dashed line. Full order model in solid line. 


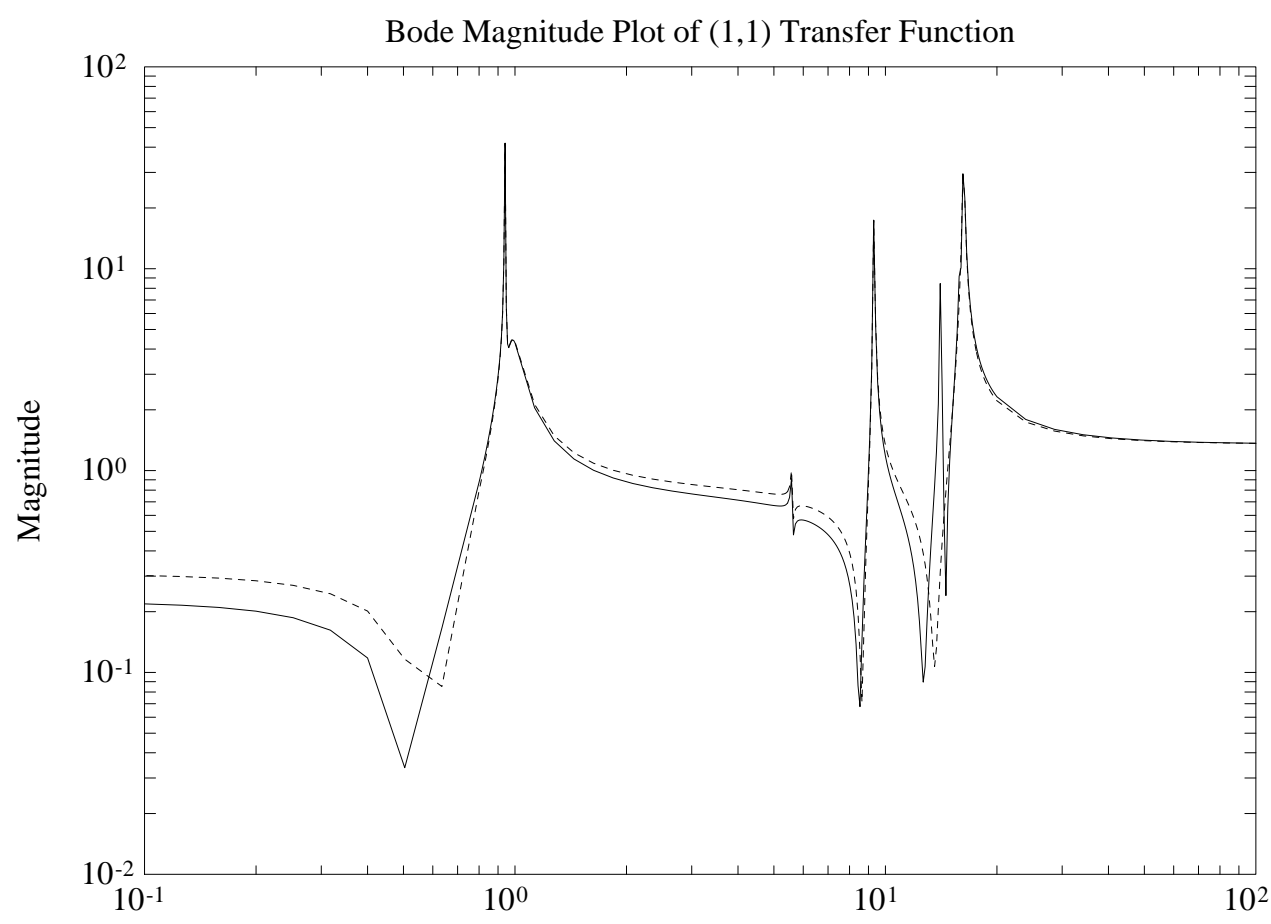

Freq. rad/s

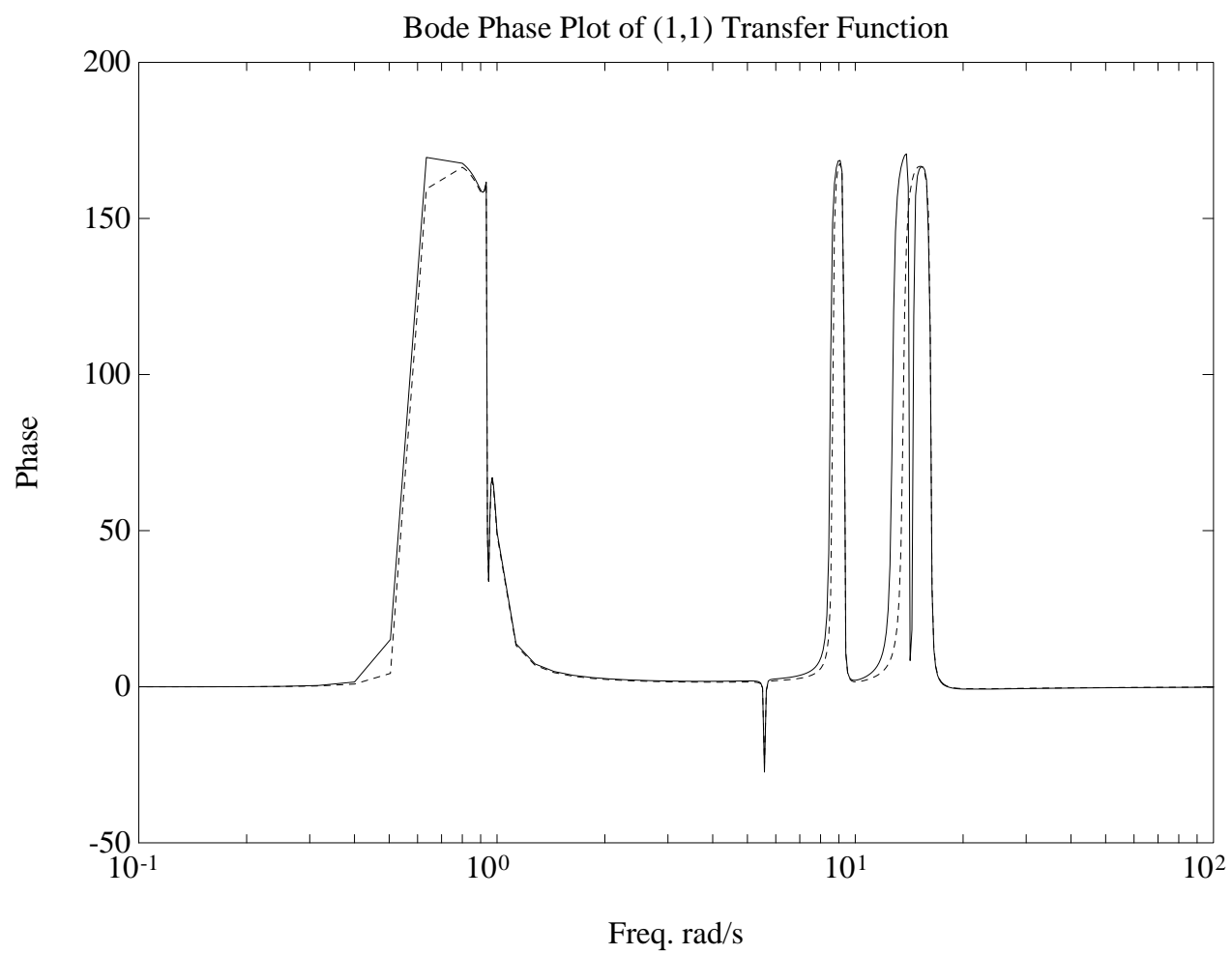

Figure 2.6 Bode Plots of Reduced Order 16 of $(1,1)$ Transfer Function; Matrix Pencil Approach in dashed line. Full order model in solid line. 


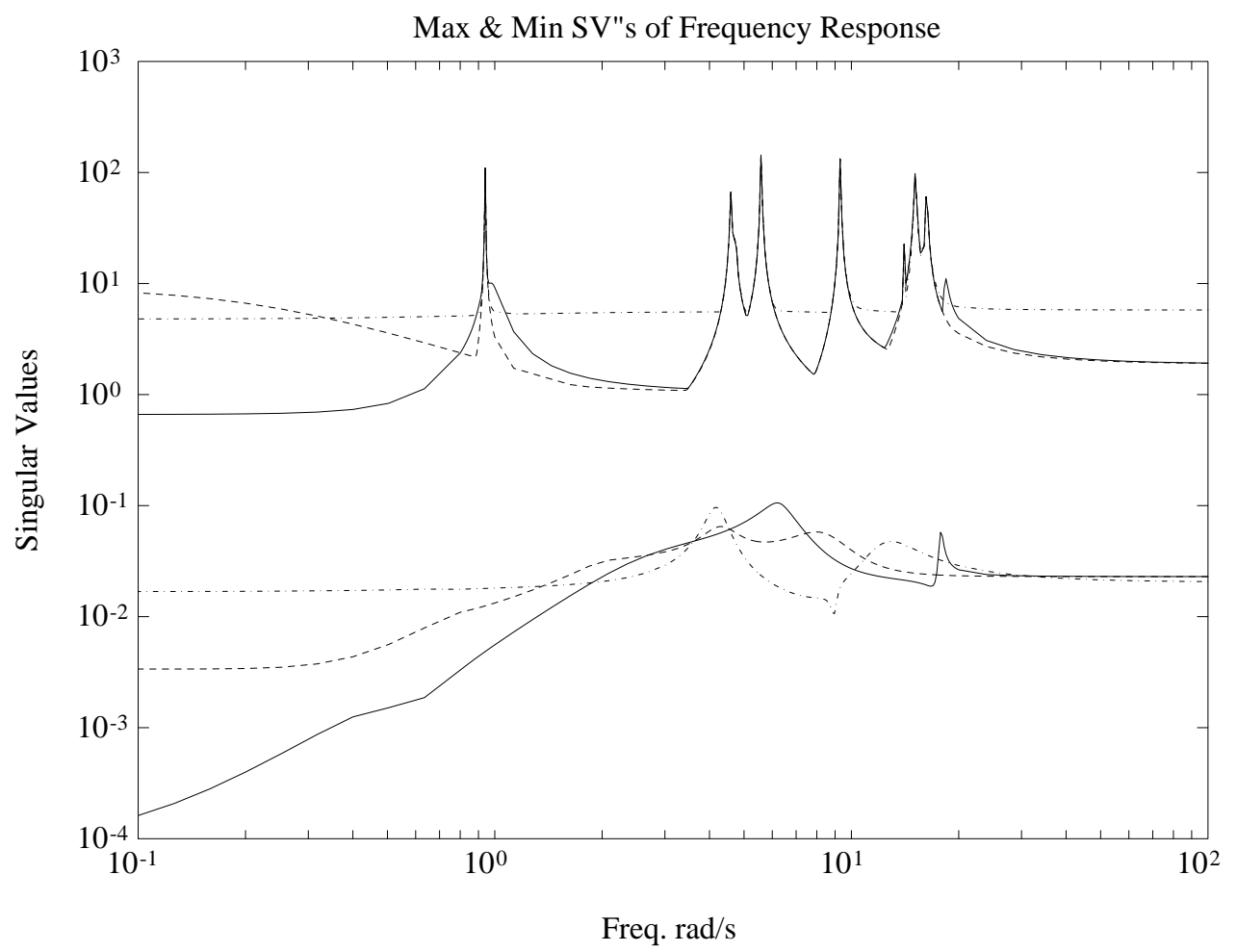

Figure 2.7 Max \& Min SV's of Reduced Order 20 Models; Balanced Realization (dashed) and Hankel Norm (dashdot) Methods. Full order model in solid line.

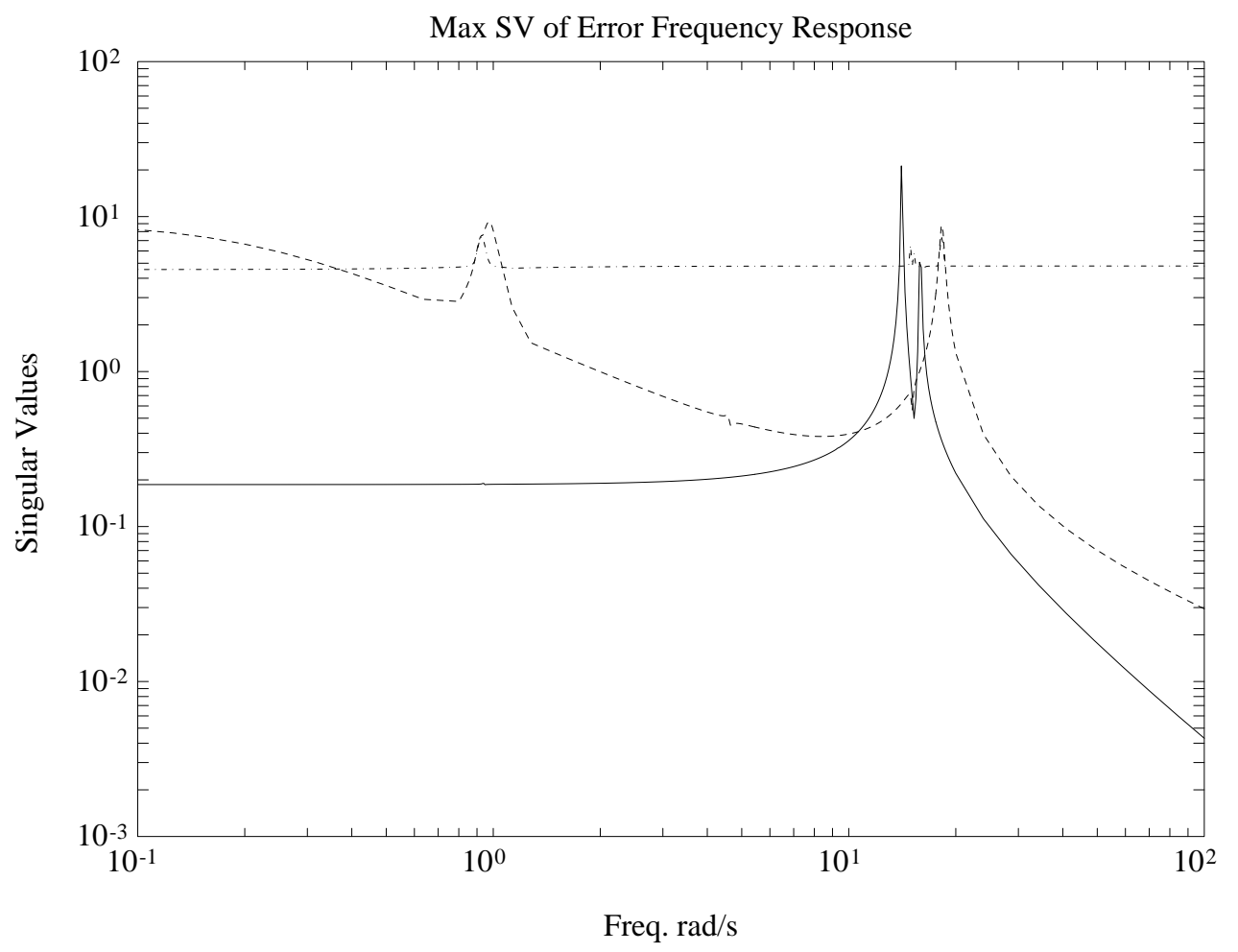

Figure 2.8 Mav SV of Error for Reduced Order 20 Models. Matrix pencil in solid line, balanced realization in dashed line, and Hankel norm in dashdot line. 


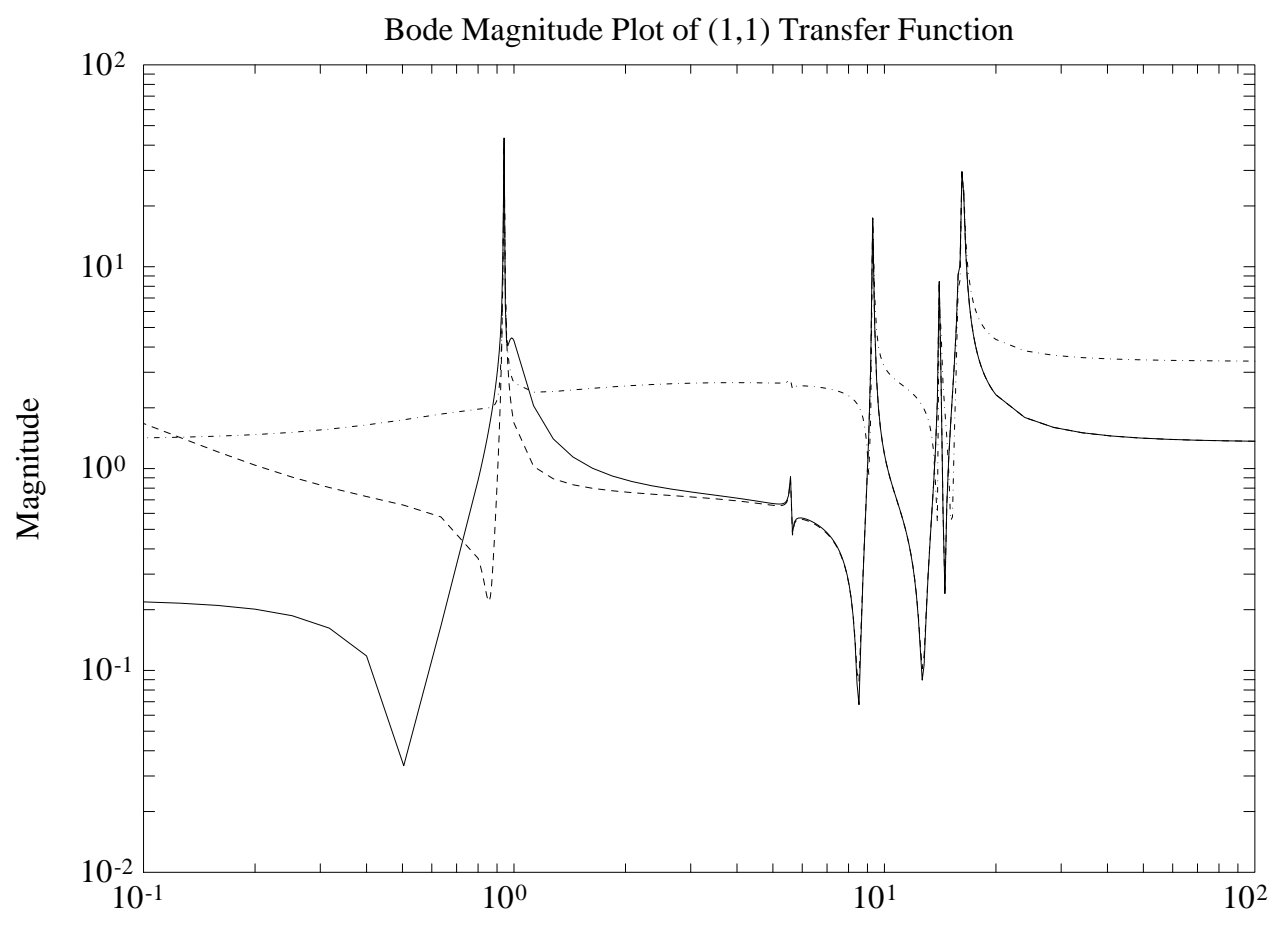

Freq. rad/s

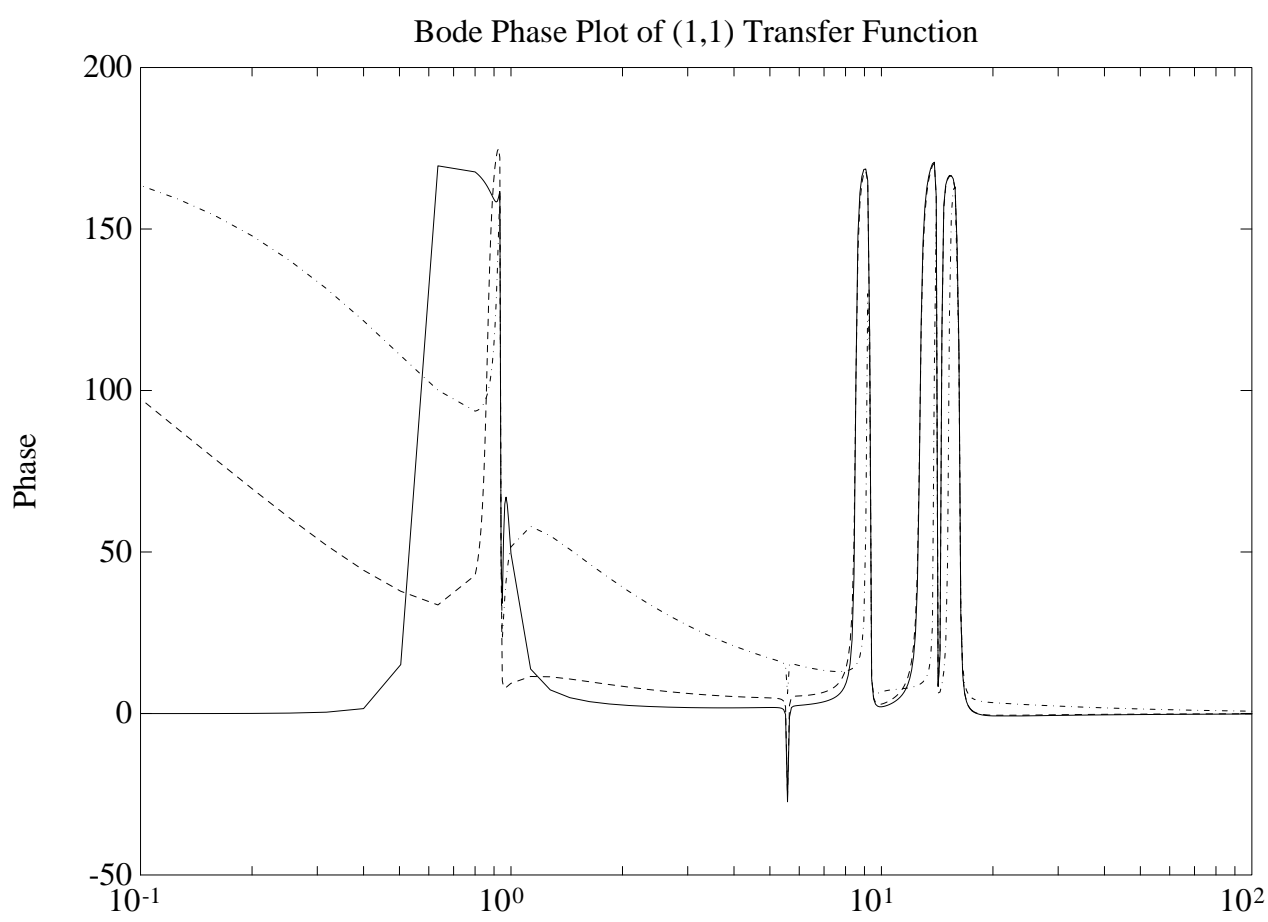

Freq. $\mathrm{rad} / \mathrm{s}$

Figure 2.9 Bode Plots of Reduced Order 20 of $(1,1)$ Transfer Function. Balanced Realization (dashed) and Hankel Norm (dashdot); full order model in solid. 


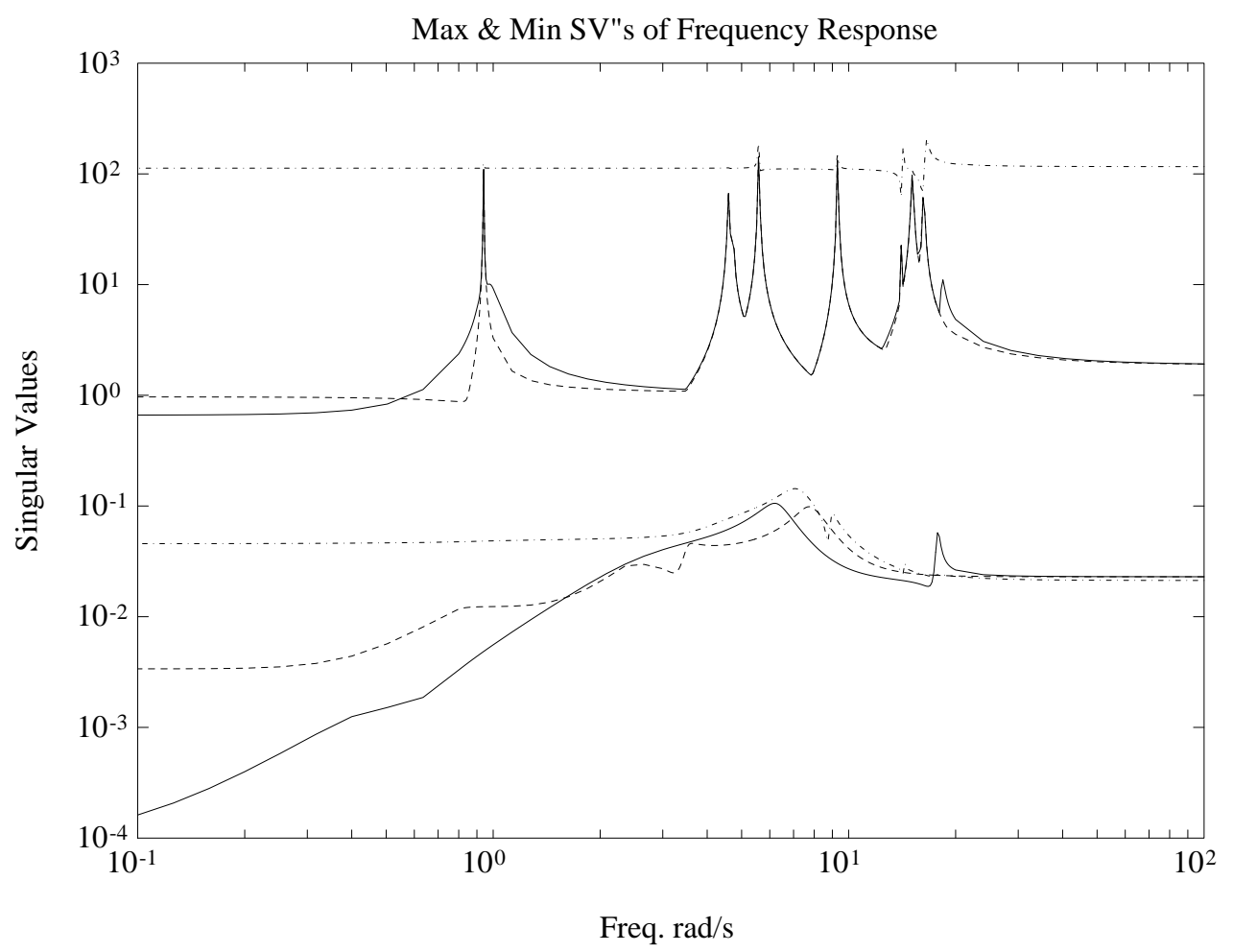

Figure 2.10 Max \& Min SV's of Reduced Order 16 Model. Balanced Realization (dashed) and Hankel Norm (dashdot) Methods; full order model in solid.

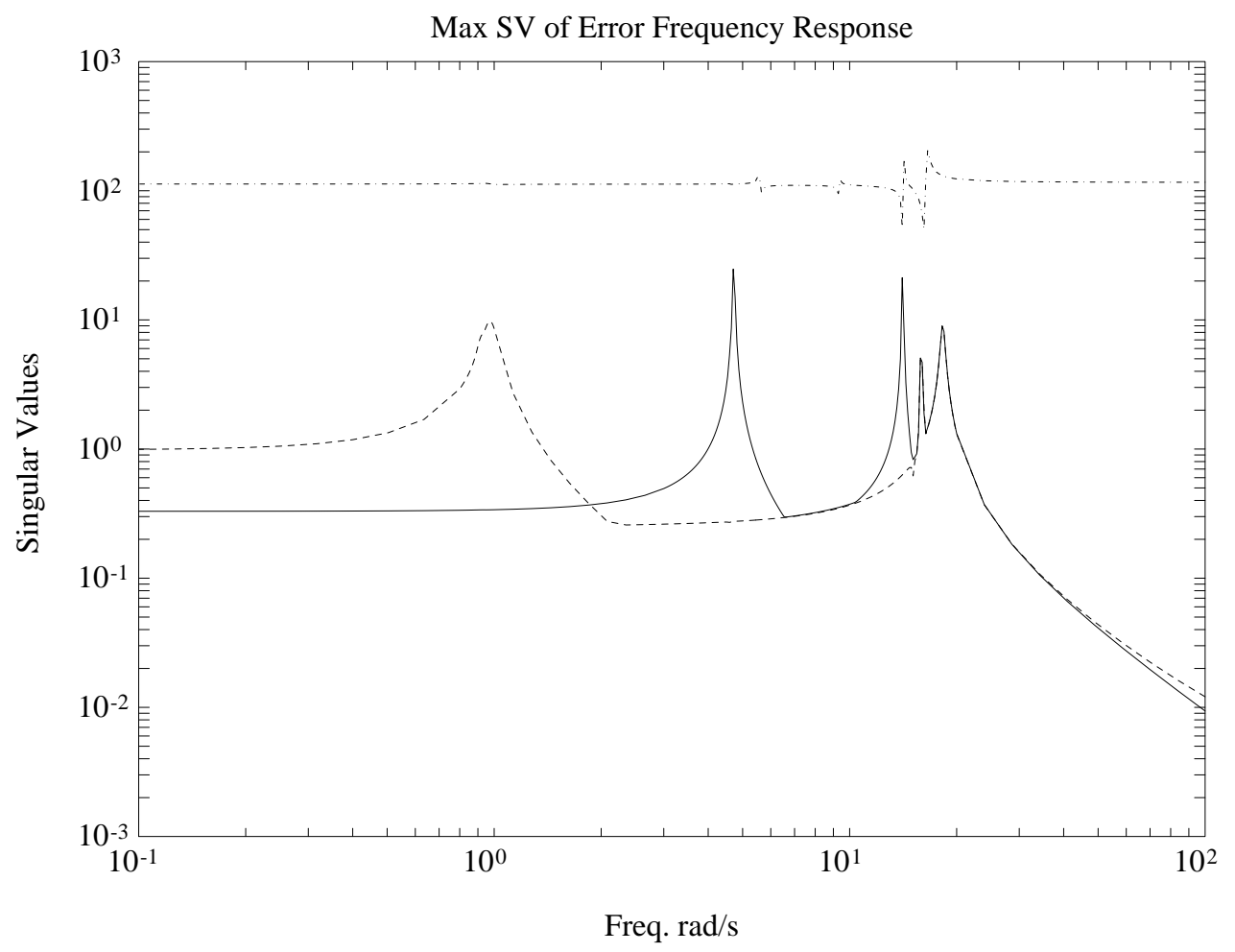

Figure 2.11 Max SV of Error for Reduced Order 16 Models. Matrix Pencil in solid line, Balanced Realization in dashed line, and Hankel norm in dahdot line. 


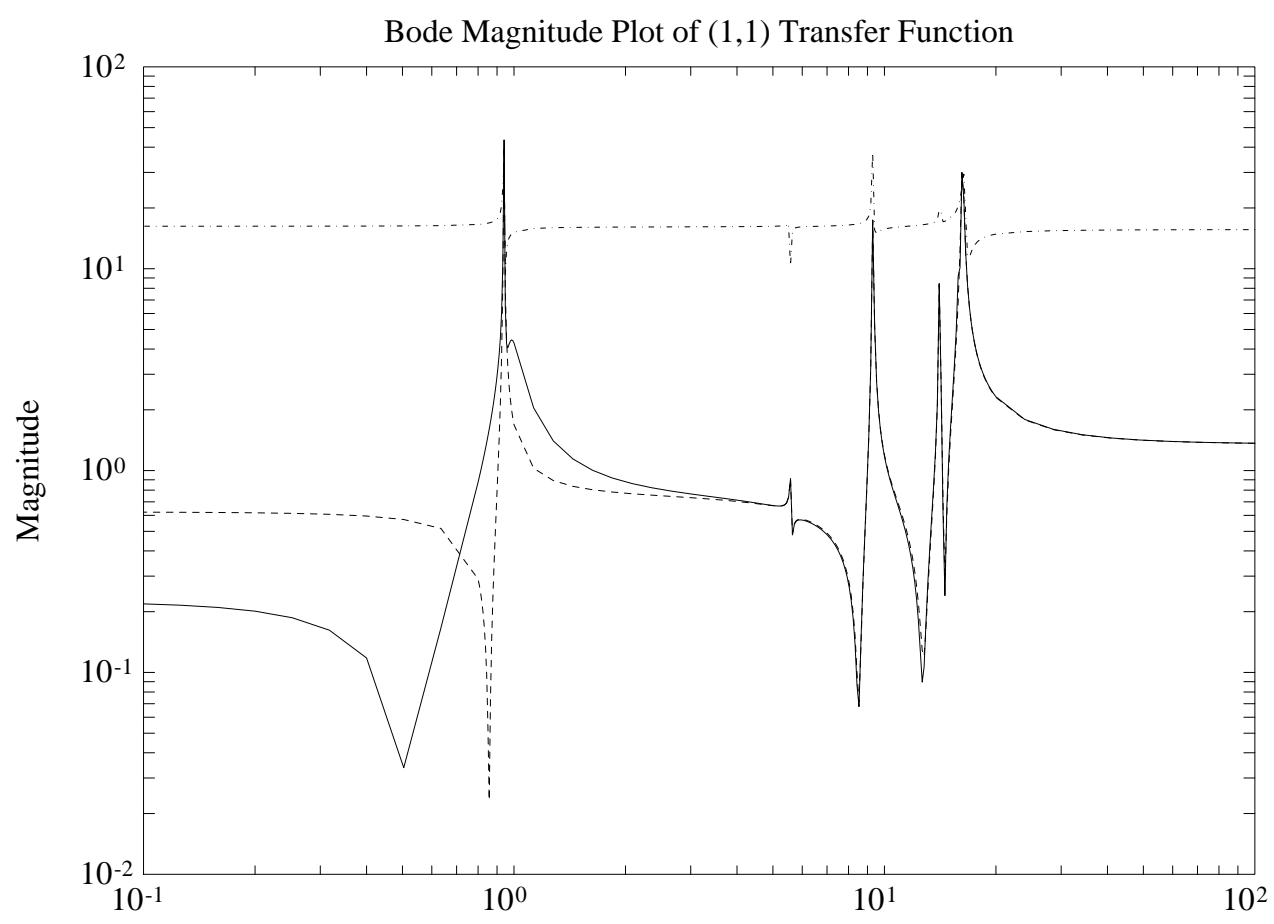

Freq. rad/s

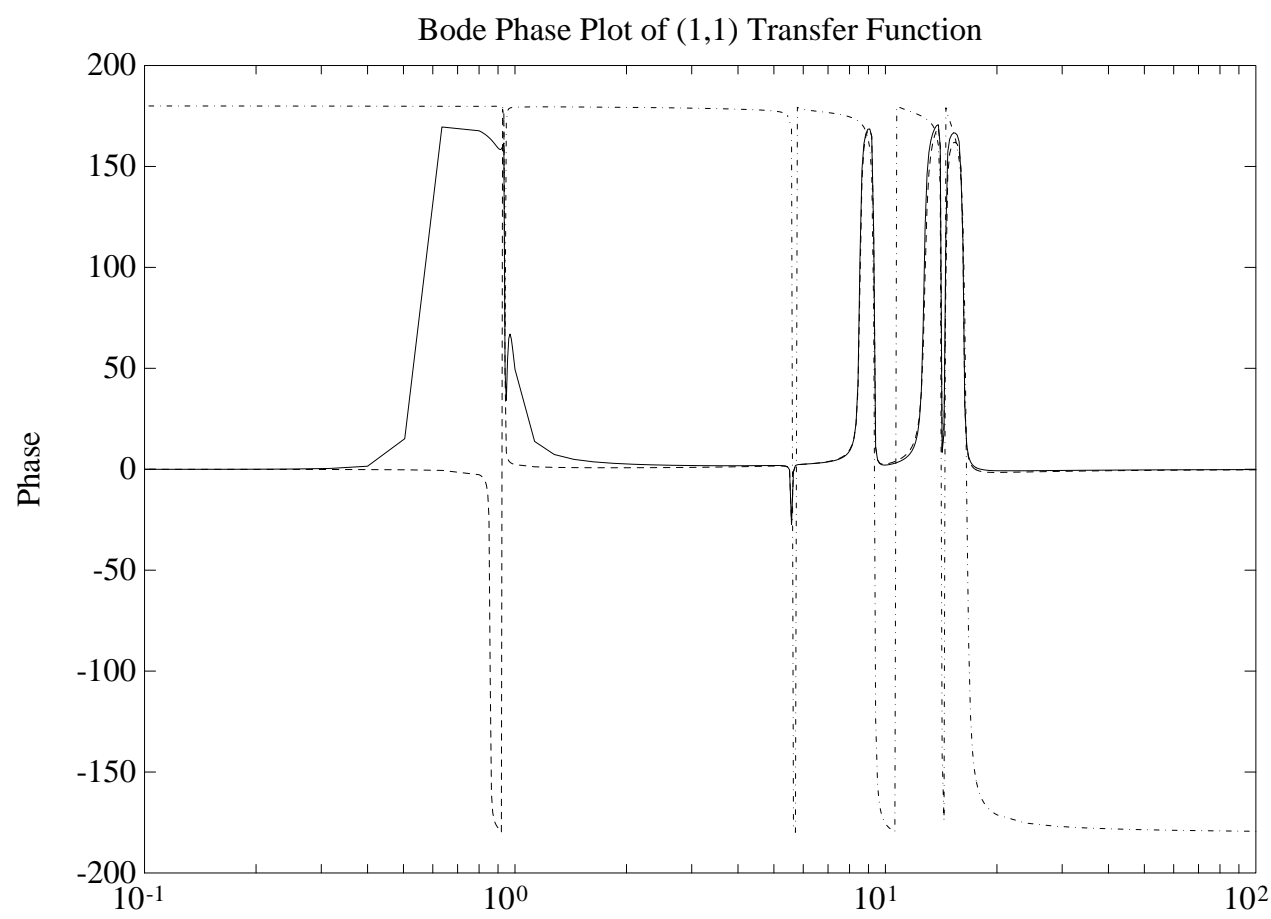

Freq. $\mathrm{rad} / \mathrm{s}$

Figure 2.12 Bode Plots of Reduced Order 16 of $(1,1)$ Transfer Function. Balanced Realization (dashed) and Hankel Norm (dashdot); full order model (solid). 